\title{
Contribution À Une Étude Floristique De « Aguelmouss-Moulay Bouaazza -Tiddass » (Plateau Central, Maroc)
}

\author{
Mustapha Hachi \\ Abdellah Rahou
}

Equipe de Biotechnologie et Valorisation des Ressources Végétales, FSM, UMI-Maroc

Kamal Lamfadal

Direction régionale des Eaux et Forêt et de la conservation des sols, Meknès doi: 10.19044/esj.2016.v12n18p267 URL:http://dx.doi.org/10.19044/esj.2016.v12n18p267

Abstract

The study of wealth and floristic diversity, conducted during March, April, May and June 2015 in the region (Aguelmous - Moulay Bouazza Oulmès- Tiddas- Maaziz) belonging to the central plateau, revealed the existence of 360 plant species; 46 species of Fabaceae, 44 Asteraceae, 32 and 19 respectively from Caryophyllaceae and Lamiaceae. These families comprise $43.9 \%$ of the entire flora of the study area, the rest of the species belong to 48 families with $56.10 \%$.

A significant number of endemic has been inventoried: 43 of Morocco, one of Morocco and the Iberian Peninsula 6 of Morocco and Algeria, 4 of Morocco, Algeria and Tunisia. Moreover, three rare and endangered species have been recorded.

Also, ecological analysis based on floristic surveys between $230 \mathrm{~m}$ and 1630 $\mathrm{m}$ shows that the altitude is a discriminating factor in the distribution of vegetation.

Keywords: Wealth, diversity, inventory, endemic floristic survey, Central Plateau, Morocco

Résumé

L'étude de la richesse et de la diversité floristique, réalisée pendant les mois de Mars, Avril, Mai et Juin 2015, dans la région (Aguelmouss Moulay Bouazza - Oulmès- Tiddas- Maaziz), relevant du plateau central a révélé l'existence de 360 espèces végétales dont 46 appartiennent à la famille des Fabacées, 44 à celle des Astéracées, 32 aux Caryophyllacées et 19 aux Lamiacées. Ces familles comptent 43,9\% de l'ensemble de la flore 
de la région étudiée, le reste des espèces, soit 56,10\% appartient à 48 familles. Un nombre important d'endémiques a été inventorié:43 endémiques du Maroc, une espèce endémique du Maroc et de la péninsule Ibérique, 6 endémiques du Maroc et de l’Algérie, 4 endémiques du Maroc, de l'Algérie et de la Tunisie. Par ailleurs, 3 espèces rares ou menacés ont été recensées. Aussi, l'analyse écologique basée sur des relevés floristiques effectués entre $230 \mathrm{~m}$ et $1630 \mathrm{~m}$ montre que l'altitude est un facteur discriminant dans la répartition des formations végétales.

Mots clés : Richesse, diversité, inventaire, endémique, relevé floristique, Plateau central, Maroc.

\section{Introduction}

La région méditerranéenne s'individualise par de nombreuses originalités physiques et écologiques qui confèrent à sa biodiversité une valeur patrimoniale mondiale.

Le royaume du Maroc couvre une superficie de $710.850 \mathrm{~km} 2$ avec $500 \mathrm{~km}$ de côte méditerranéenne et $3000 \mathrm{~km}$ de côte atlantique. Sa situation géographique et ses importantes chaînes de montagnes, l'Atlas et le Rif, lui confèrent une grande variété bioclimatique (humide à saharien) et une importante diversité biologique: 4500 taxons dont 537 endémiques (Hammoudi, 2002). Le domaine forestier marocain s'étend sur une superficie d'environ 9 millions d'hectares dont 4,5 millions d'hectares de forêts et matorrals, 3 millions d'hectares de nappes alfatières, 1 million d'hectares d'acacia sahariens et 0,5 millions d'hectares de reboisement (Hammoudi, 2002).

Les formations végétales qui constituent la forêt du plateau central présentent un intérêt multiple sur le plan biologique, économique et social. Cependant, la dégradation des forêts est un problème sérieux dans la plupart des pays en voie de développement.

A cet effet, des efforts urgents de protection doivent être entrepris afin de préserver cette richesse floristique. Néanmoins, la connaissance de la diversité des écosystèmes, sa richesse biologique et son impact sur l'équilibre socioéconomique et environnemental est une condition nécessaire pour élaborer une stratégie de conservation des ressources naturelles d'où l'intérêt d'une étude plus étendue à l'échelle régionale ou même nationale. Ainsi, le présent travail de recherche concerne essentiellement le recensement de la richesse et la diversité floristique de la région du plateau central marocain. 


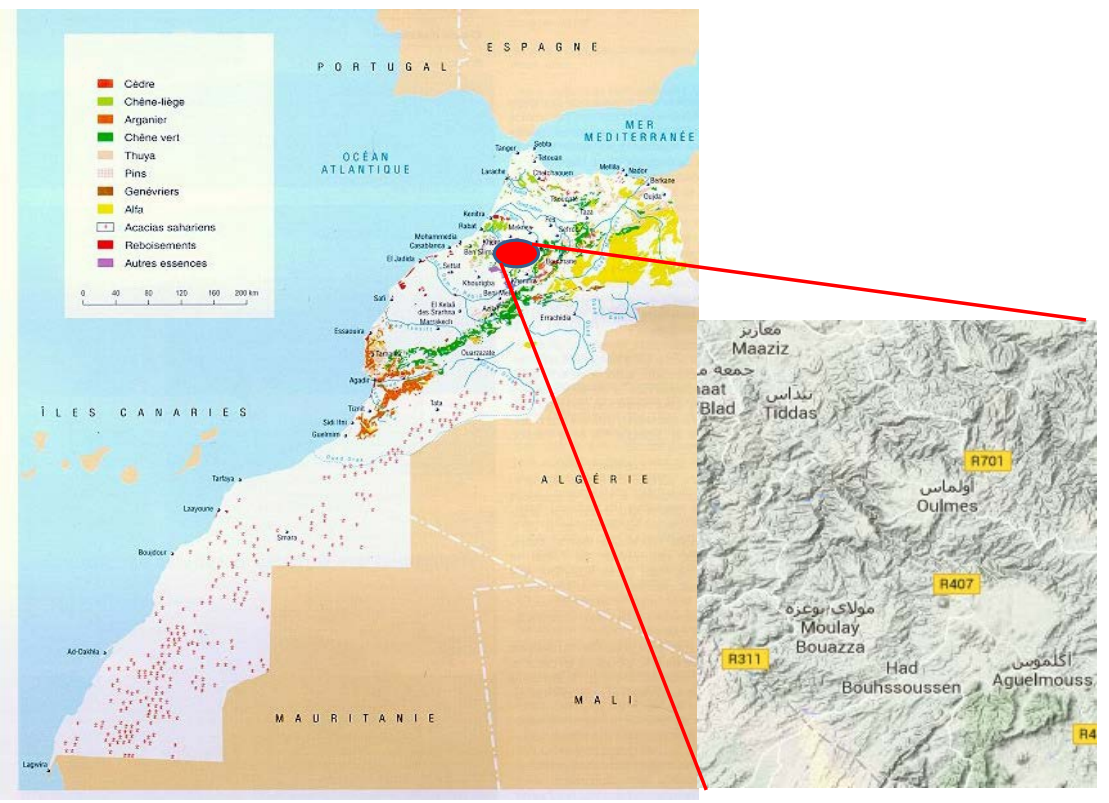

Fig 1 : Situation géographique de la région d'étude

\section{Materiel et Méthode d'étude}

\section{Présentation de la région d'étude}

La zone d'étude fait partie du plateau central marocain (Beaudet, 1969) ; elle relève en grande partie de la province de Khemisset, chevauche sur la province de Khénifra et fait partie du territoire des communes rurales de : Aguelmouss, Oulmès, Tiddas, Maaziz, Moulay Bouaâza et Had Bouhssoussen.

Elle culmine au sud-est (jbel Mtourzgene, $1630 \mathrm{~m}$ ) et retombe par une série de gradins vers le nord-ouest. Elle est coupée de nombreuses vallées dont certaines sont profondes (Oued Grou, Oued Boulahmael).

La zone d'étude caractérisée par un relief très accidenté, est constituée par des granites et des roches métamorphiques qui y affleurent essentiellement en deux massif distincts: au nord-ouest d'Aguelmouss et à l'Ouest d'Oulmès.

Les schistes primaires (Ordovicien, Silurien, Dévonien ou Carbonifère) y sont très largement représentés.

Quelques calcaires d'âge essentiellement Dévonien apparaissent dans des secteurs localisés et ne représentent que des superficies peu importantes.

Sur les formations primaires de la meseta reposent les placages plus ou moins étendus du complexe détritique permo-triasique (argiles rouge gypse et sel) dans lequel s'interstratifient des basaltes épandus en coulées que l'érosion ultérieure a plus ou moins morcelées (Combe et al.1975). 
Le climat varie avec l'altitude en basse altitude, il est semi -aride et en moyenne altitude, il est subhumide et humide.

Par ailleurs, en raison de la complexité topographique, des interférences de l'altitude, et de la continentalité sur la bioclimatologie, il est délicat de préciser dans le détail la répartition des essences. On trouve des zones forestières, et les versants des grandes vallées sont presque exclusivement le domaine du chêne-liège sur les sols siliceux, et d'oléastre, de lentisque et de Tizra sur sols calcareux ou argileux.

Aussi, le chêne vert s’y introduit souvent vert le sud- est, mais disparait vers le sud.

Le jujubier devient très abondant en approchant du plateau des phosphates (Combe et al.1975).

\section{Méthode d'étude}

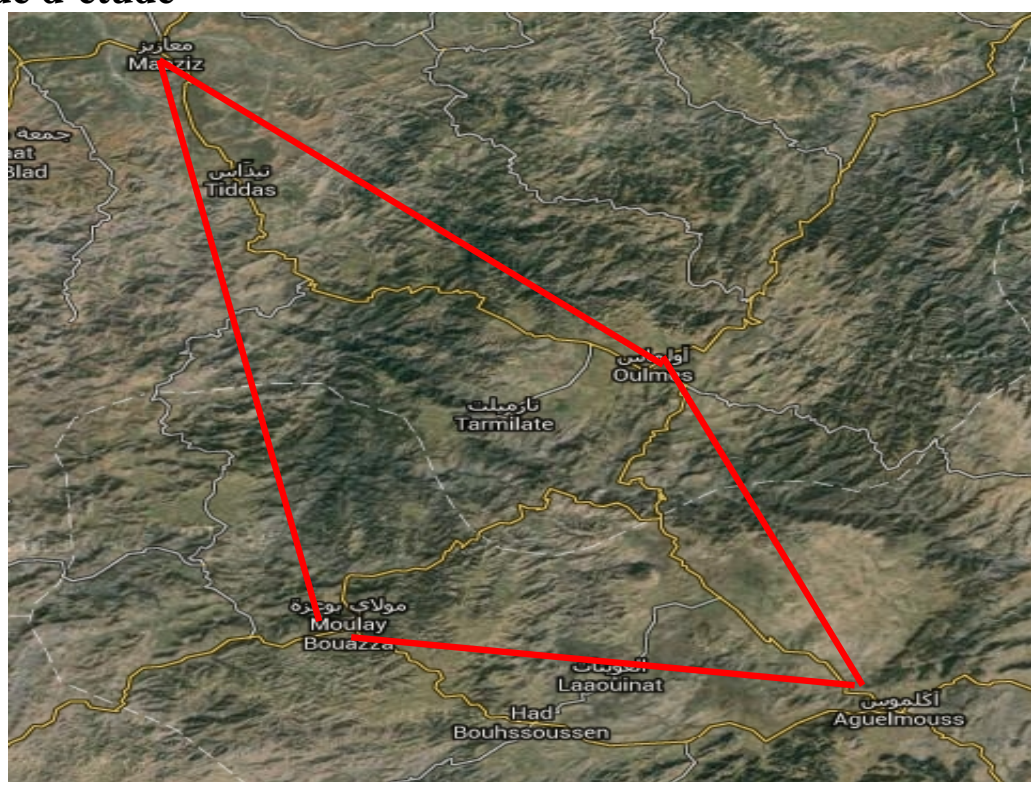

Fig 2 : situaion des transects phytoécologiques

Des campagnes ont été réalisées pendant mars, avril, mai et juin 2015 et ont permis le choix de 4 transects, de manière équilibrée et selon une stratification altitudinale entre $230 \mathrm{~m}$ et $1630 \mathrm{~m}$.

Les transects choisis sont les suivants :

\section{Transect 1}

Il est établi d'Aguelmouss jusqu'à Moulay Bouaazza entre 900m et 1630m. Les substrats géologiques sont des schistes, le bioclimat est de type semi aride et subhumide frais. 


\section{Transect 2}

Il est établi d'Aguelmouss à Oulmès.Les substrats géologiques sont des schistes et des granites.

Ce transect occupe une tranche altitudinale comprise entre $900 \mathrm{~m}$ et $1260 \mathrm{~m}$; le bioclimat est de type semi aride et subhumide à hiver frais.

\section{Transect 3}

Il est établi d’Oulmès jusqu’à Maaziz ; les substrats géologiques sont des schistes et des granites.

Ce transect occupe une tranche altitudinale comprise entre $430 \mathrm{~m}$ et 1030m, le bioclimat est de type subhumide et humide à hiver froid.

\section{Transect 4}

Ce transect est établi de Moulay Bouazza jusqu’ à Maaziz ; le substrat géologique est constitué de schistes et de granites.

Afin de répartir rationnellement les 80 relevés réalisés à travers toute la région, un plan d'échantillonnage a été établi dans le sens de Gounot (1969) et Godron (1968). Cet échantillonnage dit stratifier de type probabiliste consiste à exploiter toutes les informations acquises sur le milieu et la végétation afin de découper la zone d'étude en sous-unités homogènes qui seront échantillonnées séparément. Les critères qui entrent en jeu pour découper la zone d’étude en strates homogènes concernent généralement le climat, la pédologie et la végétation.

Des observations d'ordre floristiques et écologiques ont été inscrites. Ainsi, pour chaque espèce, il a été noté le type biologique, les références écologiques du sol (type, texture et humidité du sol), l'altitude, son biotope correspondant et son étage bioclimatique. Pour les observations impossibles de faire sur terrain, on a eu recours aux données bibliographiques qui ont été également exploitées.

C'est dans le but d'une valorisation et d'une gestion durable de la région de (Aguelmous - Moulay Bouazza - Oulmès- Tiddas- Maaziz), que cette étude de quantification de la richesse et de la diversité floristique a été menée.

\section{Abréviations utilisées :}

Bioclimat : Il est exprimé à l'aide du système classique d'Emberger. La plupart des informations sont empruntées à SAUVAGE (1960). Les étages bioclimatiques considérés sont :

$$
\begin{aligned}
& S \text { : Saharien } \\
& S H \text { : Subhumide } \\
& \text { SA: Semi-aride }
\end{aligned}
$$

$$
\begin{aligned}
& A \text { : Aride } \\
& H \text { : Humide } \\
& \text { SH: Sub- }
\end{aligned}
$$

humide

HM: Haute montagne

Type biologique: $\mathrm{Ph}$ (Phanérophytes), $\mathrm{Np}$ (Nanophanérophytes), C(Chaméphytes), H(Hémicryptophytes), G (Géophytes), Th (Thérophytes). 
Endémisme: EMA (Endémiques du Maroc et d'Algérie) ; EMI (Endémiques du Maroc et de la péninsule Ibérique); EMAT (Endémiques du Maroc, Algérie et Tunisie).

Les espèces rares ou menacées: RR: très rare (menacée); R: rare; R ?: soupçonnée rare.

Répartition géographique :

- Ms : Maroc saharien - Man : Maroc atlantique nord

- As : Atlas saharien

- Op : plateaux du

Maroc oriental

- $\quad$ AA : Anti Atlas

- Om : monts du Maroc

oriental

- $\quad$ HA : Haut Atlas

- LM : littoral de la

Méditerranée

- $\quad$ MA : Moyen Atlas

- R : Rif

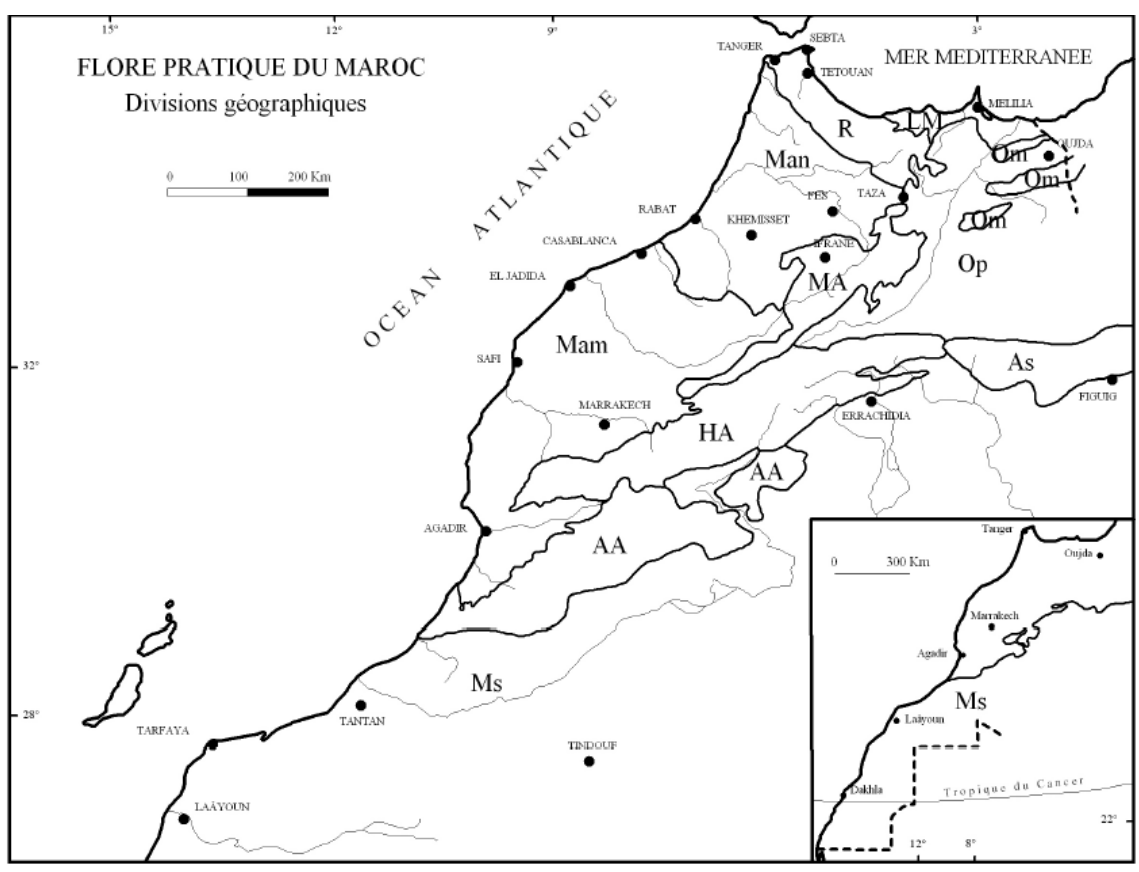

Fig3 : Carte des divisions floristiques

(Flore pratique du Maroc, Fennane M. \& M. Ibn Tattou, 1999)

Inventaire floristique :

ANACARDIACEAE

- Pistacia L.

- $\quad$ Pistacia atlantica Desf. (Btem)

$\mathrm{Ph}$ - plaines, basses et moyennes montagnes - A SA SH - As AA HA MA Mam Man Om LM R - localité : Aguelmouss (1250m), Had bouhssoussen 
(forêt sidi çalah ,1450m), Moulay Bouaazza (1040m), Mbarkyene (820m), Jbel el Harch (930m), Al Go-Ida (1450m), Oulmès (1170m) ;

- $\quad$ Pistacia lentiscus L. (Drou)

$\mathrm{Ph}$ - plaines, basses et moyennes montagnes - SA SH H - As AA HA MA Mam Man Om LM R - localité : Toute la zone d'étude.

- Rhus L.

- $\quad$ Rhus pentaphylla (Jacq.) Desf.

Nph ; Plaines et basses montagnes ; SA SH ; toutes les divisions du pays ; localité : toute la zone d'étude.

ARALIACEAE

- Hedera L.

- Hedera helix L.

$\mathrm{Ph}$ - roches ombragés des forêts et matorrals des plaines, basses et moyennes montagnes - SA SH H - AA HA MA Man LM R - localité : Aguelmouss (Torziane 1630m), Jbel sidi çalah (1415m), Massif Amadghouss (1540m), Algo'Ida (1475 m)

ARACEAE

- $\quad$ Arisarum vulgare Targ. Tozz. (Capuchon de Moine)

G - Habites les lieux ombragés et les pâturages rocailleux - SH H - AA HA MA Mam Mam Op Om - localité : Large distribution géographique au niveau de la zone d'étude.

\section{ARISTOLOCHIACEAE}

- $\quad$ Aristolochia L. (berztam)

- $\quad$ Aristolochia paucinervis Pomel

G - Champs, ermes, matorrals ; plaines, basses et moyennes montagnes -SA SH H - AA HA MA Mam Man Om Op LM R- localité : Large distribution géographique au niveau de la zone d'étude.

- $\quad$ Aristolochia boetica L.

$\mathrm{Ph}$ - Rochers, broussailles et forêts ; plaines et basses montagnes - SA SH H - AA HA Mam Man Om Op LM R- localité : Large distribution géographique au niveau de la zone d'étude.

\section{APOCYNACEAE}

- $\quad$ Nerium oleander L. (Laurier rose)

Nph - bords des oueds et cours d'eaux et des milieux humides - SA SH H tout le Maroc; localité : Toute la zone d'étude surtout dans les lieux humides.

\section{ASTERACEAE (Composées)}

\section{- Anacyclus clavatus Desf.}

Th - SH H - broussailles rocailleuses, pâturages, champs et forêt claires - Ms MA Mam Man Op Om LM R- EM - localité : Toute la zone d'étude.

- $\quad$ Anacyclus radiatus loisel. 
Th - pâturages et forêt claires - SH - Ms MA Mam Man - localité : Toute la zone d'étude.

\section{- $\quad$ Andryala arenaria Boisier \& Reuter}

Th - clairières des forêts - H - MA Mam Man Om LM R - EMI - localité : Toute la zone d'étude.

\section{- $\quad$ Andryala integrifolia $L$.}

Th - pâturages Clairières des forêts - AA HA MA Mam Man Op Om LM R - localité : toute la zone d'étude - EM ;

- $\quad$ Andryala laxiflora (Salzm.) Maire

Th - champs et Pâturages rocailleux - MA Mam Man - localité : toute la zone d'étude - EM ;

- $\quad$ Anthemis pedunculatus Desf.

Ch - forêts, broussailles et pâturages - SH H- As AA HA MA Mam Man Om LM R - localité : toute la zone d'étude ;

- $\quad$ Asteriscus aquaticus Less.

Th- pâturages humides - SH - HA MA Man Op Om LM R - localité : toute la zone d'étude ;

- $\quad$ Asteriscus maritimus Less.

Ch - pâturages humides et rochers - SH - HA MA Man Op Om LM Rlocalité ; toute la zone d'étude ;

- $\quad$ Asteriscus spinosus L. Schultz Bip.

$\mathrm{H}$ - terrains incultes et pierreux ; SA SH H - HA MA Man Op Om LM R localité : Toute la zone d'étude ;

- Bellis sylvestris Cyr. (Pâquerette)

$\mathrm{H}$ - pâturages et forêts - SH H - AA HA MA Mam Man Om LM R localité : Aguelmouss (1630m), Algo’ida (1475 m), Massif Amedghous (1540m), jbel Al harcha (970m) et Oulmès (1170m);

- Calendula aegyptiaca Desf.

Th - pâturages et clairières des forêts - SH - Ms MA Mam Op R - localité : Toute la zone et ne dépasse pas $1200 \mathrm{~m}$.

- $\quad$ Calendula arvensis L. (Fiori) Lanza.

Th - Champs, lieux incultes et pâturages pierreux - SH - Ms MA Mam LM localité : Aguelmouss (1100m), Ment (1200m), Oulmès (1170m) - RR.

- Calendula algeriensis Boiss.

Th - pâturages, champs et lieux incultes - SH - Ms MA Mam LM - localité : large distribution au niveau de la zone d'étude ;

\section{- Carlina hispanica $\mathbf{L}$.}

$\mathrm{H}$ - pâturages, champs et lieux incultes - SA SH H -AA HA MA Op Om LM

$\mathrm{R}$ - localité : Toute la zone d'étude et ne dépasse pas $1300 \mathrm{~m}$;

\section{- Carlina involucrata Poiret.}


Th - pâturages, champs et lieux incultes - SA SH H - AA HA MA Op Om LM R - localité : large distribution au niveau de la zone d'étude ;

\section{- Carlina racemosa $L$.}

H - pâturages, champs - SA SH H; HA MA Mam Man Om LM R localité : toute la région d'étude et ne dépasse pas 1400m.

\section{- Cardus pycnocephalus $L$.}

Th - pâturages et forêts claires - SH - MA Mam Man 0p Om LM R localité : toute la région d'étude et ne dépasse pas 1300m.

\section{- $\quad$ Centaurea pullata $L$.}

H - pâturages et clairières des forêts - SH H - AA HA MA Mam Man Om LM R - localité : toute la région d’étude et ne dépasse pas 1300m - EM

\section{- $\quad$ Centaurea calcitrapa $L$.}

H - pâturages et clairières des forêts - SASH H - AA HA MA Mam Man Om LM R - localité : Toute la région d'étude et ne dépasse pas 1300m.

\section{- $\quad$ Centaurea melitensis $L$.}

Th - Pâturages, champs et clairières des forêts - SA SH H - AA HA MA Mam Man Om LM R - localité : toute la région d’étude et ne dépasse pas 1300m

\section{- $\quad$ Chrysanthemum segetum L.}

Th - pâturages, rocailleux de montagnes - SA SH H - AA HA MA Mam Man Om LM R - localité : Toute la zone d'étude et ne dépasse pas 1200m;

\section{- Cirsium echinatum (Desf.) DC.}

H - Forêts, Pâturages argileux ou pierreux - SH H - AA HA MA Man Om LM R -localité : Toute la zone d'étude et ne dépasse pas 1200m;

- Crepis vesicaria $L$.

(H)(Th) - Broussailles, pâturages et champs - AA HA MA Mam Man Op Om LM R -localité : Toute la zone d'étude et ne dépasse pas 1200m - EM

\section{- $\quad$ Crepis pulchra $L$.}

Th - Pâturages rocailleux - SH - AA HA MA Mam R - localité : Mtourzgene (1630m) ; Algo’ida (1475 m) ; Ait ikkou (1052 m) ; jbel Al harcha (970m); Jbel bou Nzahl (1048 m) - EMA.

\section{- $\quad$ Cynara cardunculus $L$.}

G - Champs et pasturages - H - MA Mam Man Op Om R - localité : large distribution et ne dépasse pas 1300m ;

\section{- $\quad$ Cynara humilis L.}

G - pâturages des terrains calcaires et siliceux - H - HA MA Mam Man Rlocalité : large distribution et ne dépasse pas 1300m ;

\section{- $\quad$ Evax pygmaea L. Brot.}

Th - SA SH H - AA HA MA Mam Man Om LM R - localité : large distribution au niveau de a zone d'étude et ne dépasse pas $1200 \mathrm{~m}$;

\section{- $\quad$ Filago gallica $L$.}


Th - Champs, pâturages et forêts claires - SH H - AA HA MA Mam Man Om LM R - localité : large distribution dans la zone d'étude et ne dépasse pas $1200 \mathrm{~m}$;

\section{- $\quad$ Filago heterantha Gauss.}

Th - Pâturages rocailleux et clairières des forêts - SH H - As HA MA Mam R - localité : large distribution dans la zone d'étude et ne dépasse pas $1200 \mathrm{~m}$;

- $\quad$ Hyoseris radiata $L$.

$\mathrm{H}$ - Rochers, rocailles et clairières des forêts - SH H - AA HA MA Mam Man Om LM R - localité : large distribution dans la zone d'étude ;

\section{- Hyoseris scabra $L$.}

H - Rochers, rocailles et pâtura ges humides - SA SH - HA MA Mam Man Op Om LM R - localité : large distribution et ne dépasse pas 1200m ;

\section{- Hypochoeris achyrophus L.}

Th - Rochers, pâturages et clairières des forêts - SH H - HA MA Mam Man Om Op LM R - localité : large distribution et ne dépasse pas 1200m ;

\section{- Hypochoeris glabra L.}

Th - Rochers, pâturages et clairières des forêts - SH H - As HA MA Mam Man Om Op LM R- localité : large distribution et ne dépasse pas 1200m ;

- Hypochaeris laevigata (L.) Ces., Pass. \& Gibelli

H - Rochers des montagnes - SH H - HA MA Man LM R- localité : large distribution et ne dépasse pas $1200 \mathrm{~m}$;

- $\quad$ Hypochaeris radicata $L$.

$\mathrm{H}$ - pâturages et clairières des forêts - SH H - HA MA Mam Man Om LM $\mathrm{R}$ - localité : large distribution et ne dépasse pas $1200 \mathrm{~m}$;

- Leuzea conifera (L.) DC.

H - Forêts et broussailles de montagne s - SH H - HA MA Om R - localité : forêt sidi Hssaine $(1200 \mathrm{~m})$, Ment $(1214 \mathrm{~m})$, Oulmès $(1170 \mathrm{~m})$, Maaziz (430m), Aguelmouss (Jbel Torziane 1630m) , Moulay bouaaza (930m);

\section{- $\quad$ Mycelis muralis (L.) Gaertn}

Th - Bords des eaux et lieux ombragés - SA SH - localité : large distribution dans la zone d'étude ;

\section{- $\quad$ Echinops spinosus $L$.}

Th - Pâturages, plaines et clairières des forêts - SH H - AA HA MA Mam Man Op Om - localité : Toute la région d'étude ;

\section{- $\quad$ Picris hieracioides $\underline{L}$.}

H - Pâturages, plaines, matorrals et clairières des forêts - SH H - AA HA MA Mam Man Op Om - localité : large distribution et ne dépasse pas 1200m;

\section{- $\quad$ Scolymus hispanicus L.}


H - Culture et pâturages - SA SH - Toutes les divisions géographiques du Maroc - localité : large distribution dans la zone d'étude et ne dépasse pas $1200 \mathrm{~m}$;

\section{- $\quad$ Senecio lividus $L$.}

Th - Culture et Pâturages, broussailles et forêts - SA SH - HA MA Man R localité : large distribution dans la zone d'étude et ne dépasse pas $1200 \mathrm{~m}$;

- $\quad$ Silybum marianum ( $\underline{\text { L. }}$ Gaertn.

Th - SH H HA MA Mam Man Op Om - localité : large distribution dans la zone d'étude et ne dépasse pas $1200 \mathrm{~m}$;

- $\quad$ Tolpis barbata (L.) Gaerther

Th - Pâturages et forêts claires - SH H - AA HA MA Mam Man Op Om LM R - localité : large distribution dans la zone d'étude et ne dépasse pas $1200 \mathrm{~m}-\mathrm{EM}$.

\section{- $\quad$ Tolpis umbellate Bertol.}

Th - Pâturages et forêts claires - SH H - MA Mam Man Om LM R - localité : large distribution dans la zone d'étude et ne dépasse pas 1200m ;

\section{BERBERIDACEAE}

\section{- Berberis hispanica Boiss.}

Nph (Ph) - Forêts, Matorrals, fissures de rochers des montagnes - SH H As HA MA R - Localité : Aguelmouss (1250m); Al go-Ida (1450m);

\section{BORAGINACEAE}

\section{- Borago L.}

\section{- $\quad$ Borago officinalis $L$.}

Th - lieux incultes ; plaines et basses montagnes - SA SH H - AA HA MA Mam Man Op Om LM R - Localité : Toutes la zone d'étude ;

\section{- $\quad$ Cerinthe L.}

\section{- $\quad$ Cerinthe major $L$.}

Th - forêts et matorrals dégradés, ermes, cultures, jachères ; plaines et moyennes montagnes - A Sad Saf SH H - Maroc non saharien

\section{- $\quad$ Echium L.}

\section{- $\quad$ Echium horridium Batt.}

Th - rochers, rocailles, plaines et basses montagnes - SA - Ms AA HA Mam Man Op LM R - Localité : toute la zone d'étude ;

\section{- $\quad$ Echium plantagineum}

Th - milieux ouverts, terrains ; plaines et basses montagnes - A SA SH H Maroc non saharien

\section{- $\quad$ Echium humile Desf.}

$\mathrm{H}(\mathrm{Th})$ - forêts et matorrals dégradés, ermes, steppes ; plaines, basses et moyennes montagnes - SA SH H - Tout le Maroc - EM

CALLITRICHACEAE :

\section{-Callitriche L.}


- Callitriche lusitania Schotsman.

$\mathrm{H}$ - eaux stagnantes ou à courant très lent - SA - Mam Man - Localité : Jbel el harcha $(930 \mathrm{~m})$, oulmès $(1170 \mathrm{~m})$ et Tiddass $(530 \mathrm{~m})$;

- Callitriche mathezzi Schotsman.

H - lieux inondés - SA SH - Man - Localité : Aguelmouss (1170m), Maaziz (430m) et ne dépasse pas 1500m - EM ;

- $\quad$ Callitriche Stagnalis Scop.

$\mathrm{H}$ - Eaux stagnantes ou à écoulement très lent, rives vaseuses ; plaines et basses montagnes - A SA SH H - AA HA MA Mam Man Op Om LM R localité : Oulmès (1170m) ;

CARYOPHYLLACEAE

- Arenaria L.

- $\quad$ Arenaria emarginata Brot.

Th- Terrains sablonneux de couverts, plaines et basses montagnes - A SA SH H - AA HA MA Mam Man Om LM R - localité : Toute la zone d’étude - EM

- Cerastium L.

- Cerastium glomeratum Thuill.

Th - Matorrals, pâturages rocailleux ou sablonneux - A SA SH H - localité : Tout le Maroc sauf Ms

- Cerastium brachypetalum Pers

Th - forêts et pasturages des montagnes - SA SH H - HA MA Mam Man R - localité : Toute la zone d'étude ;

- $\quad$ Ceratium pentadrum $L$.

Th - pâturages sablonneux et terreux ; plaines et basses montagnes - SA SH H - MA Mam Man LM R - localité : Tidass (430m), Maaziz (520m), Oulmès (1170m) ;

- $\quad$ Ceratium atlanticum Durieu.

Th $(\mathrm{H})$ - Ruisseaux des basses et moyennes montagnes - SH H - AA HA MA Mam Man R - EM - localité : Tidass (430m), Maaziz (520m), Oulmès (1170m) ;

\section{- Herniaria L.}

- $\quad$ Herniaria lenticulata L. (herrast lahjar)

Th- lieux incultes, champs sablonneux des plaines et des basses montagnes SA SH H - localité : Tout le Maroc.

\section{- $\quad$ Holosteum L. \\ - Holosteum umbellatum L.}

Th - Cultures, ermes pierreux et sablonneux des montagnes ne dépasse pas 2300m - SA SH H - As AA HA MA Man LM R - localité : Aguelmouss (1250m), Had bouhssoussen (forêt sidi çalah ,1450m)

Paronychia Miller. 
- $\quad$ Paronychia echimulata Chater.

Th - clairières des forêts, broussailles, ermes sablonneux ; plaines et basses montagnes - SA SH - AA MA Mam Man LM R - localité : Aguelmouss (1120m) ; forêt sidi Hssein (1350m) ;

- Paronychia chlorothyrsa Murb.

$\mathrm{H}$ - Rochers et ermes rocailleux ; basses et moyennes montagnes, ne dépasse pas 2000m - A SA SH - localité : Tout le Maroc

- Paronychia capitata (L.) Lam

$\mathrm{H}$ - Rochers et ermes rocailleux, plaines, basses et moyennes montagnes - A SA SH - EM- localité : Tout le Maroc sauf le Ms

- $\quad$ Petrorhagia (Ser.) Link.

- $\quad$ Petrorhagia prolifera $L$.

Th - Clairières et pâturages ; plaines, basses et moyennes montagnes - SA SH H - HA MA Mam Man R - localité : Tidass (430m), Maaziz (520m), Oulmès (1170m) ;

- $\quad$ Petrorhagia velutina Guss.

Th - Clairières des forêts et pâturages des plaines, basses et moyennes montagnes - SA SH H - AA HA MA Mam Man Om LM R - localité : Tidass (430m), Maaziz (520m), Oulmès (1170m) ;

- $\quad$ Petrorhagia nanteuilli Burnat.

Th - Clairières des forêts et pâturages des plaines, basses et moyennes montagnes - SH H - AA HA MA Man - localité : Tidass (430m), Maaziz (520m), Oulmès $(1170 \mathrm{~m})$;

- Polycarpon L.

- $\quad$ Polycarpon sauvagei Mathez.

H (Ch) - SH - Man - localité : toute la région d'étude - EM

- Polycarpon polycarpoides Jahandiez \& Maire

$\mathrm{H}$ - Terrains rocheux, pierreux ou graveleux, surtout basses et moyennes montagnes - SA SH H - As AA HA MA Om LM R- localité : Aguelmouss (1250m), Had bouhssoussen (forêt sidi çalah ,1450m);

- $\quad$ Polycarpon tetraphyllum $\mathbf{L}$.

Th - plaines, basses et moyennes montagnes - SA SH H - Ms AA HA MA Mam Om LM R - localité : Aguelmouss (1250m), Had bouhssoussen (forêt sidi çalah ,1450m) ; Moulay Bouaazza (1040m), Mbarkyene (820m) ;

- $\quad$ Stellaria L.

- $\quad$ Stellaria alsine Grinum.

G - ruisselets et sources des montagnes - SA SH H - AA HA Mam Man R

Localité : Ment et jbel el harcha (930);

- Sagina L.

- $\quad$ Sagina apetala Ard. 
Th - Terrains incultes surtout sablonneux - A SA SH H - localité : Tout le Maroc sauf Ms.

\section{- Silene L.}

- $\quad$ Silene vulgaris (Moeuh) Garcke.

$\mathrm{G}$ - forêt claires, rocailles, champs cultivès ou non ; plaines, basses et moyennes montagnes - A SA SH H -localité : Tout le Maroc

- $\quad$ Silene mentagensis Cosson.

H (Ch) - Paturages rocailleux des basses montagnes - SA SH - AA HA MA Mam Man - EM- Localité : Oulmès (1170m) et Aguelmouss (1100m) ;

\section{- $\quad$ Silene latifolia Poiret.}

$\mathrm{H}$ - forêts, matorrals, rochers et ombragés ; plaines, basses et moyennes montagnes - SA SH H - HA MA Man Mam Om LM R - localité : Aguelmouss (1250m), Had bouhssoussen (forêt sidi çalah ,1450m) ; Moulay Bouaazza (1040m), Mbarkyene (820m) ;

\section{- $\quad$ Silene patula Desf.}

$\mathrm{H}$, (Ch)- rochers, forêts, rocailles ombragés des basses et moyennes montagnes - SA SH H - HA MA Man Mam Om LM R - Localité : Jbel el Harch (930m) - EMA

\section{- $\quad$ Silene coelirosa (L.)Godron}

Th - forêts claires, matorrals et pâturages ; plaines et montagnes - SA SH HMA Mam Man Om LM R - localité : Aguelmouss (1250m), Had bouhssoussen (forêt sidi çalah ,1450m), Moulay Bouaazza (1040m), Mbarkyene (820m), Jbel el Harch (930m), Al Go-Ida (1450m) ;

\section{- $\quad$ Silene conica $L$.}

Th- matorrals, pâturages, pierreux et sableux ; plaines, basses et moyennes montagnes - SA SH H- As AA HA MA Om LM R - localité : Aguelmouss (1250m), Had bouhssoussen (forêt sidi çalah ,1450m), Moulay Bouaazza (1040m), Mbarkyene (820m);

\section{- $\quad$ Silene muscipula $L$.}

Th - Champs et pâturages rocailleux; plaines, basses et moyennes montagnes - A SA SH - Ms AA HA MA Mam Man Op R - localité : Aguelmouss (1250m), Had bouhssoussen (forêt sidi çalah ,1450m), Moulay Bouaazza (1040m), Mbarkyene (820m) ;

\section{- $\quad$ Silene apetulla Willd.}

Th - Moissons, champs incultes, terrains sablonneux ou pierreux, plaines et basses - A SA H - AA HA Mam Man Op Om R - localité : Moulay Bouaazza (1040m), Mbarkyene (820m)

\section{- $\quad$ Silene secundiflora Otth.}

Th - Rochers, rocailles, matorrals ; plaines et basses montagnes - SA SH localité : Tout le Maroc.

\section{- $\quad$ Silene nocturna $L$.}


Th - Clairières des forêts et pâturages; plaines basses et moyennes montagnes - A SA SH - localité : Tout le Maroc sauf Ms.

\section{- $\quad$ Silene gallica $L$.}

Th - pâturages sablonneux et pierreux des plaines, basses et moyennes montagnes - SA SH H - localité : Tout le Maroc sauf Ms et As

- $\quad$ Silene tridentata Desf.

Th - pâturages rocailleux; plaines et basses montagnes - A SA SH localité : Tout le Maroc sauf Ms.

\section{- $\quad$ Silene pomellii Batt.}

Th - champs pierreux, pâturages rocailleux ; plaines et basses montagnes SA SH H - AA HA MA Mam Man Om LM R - EMA - localité : Aguelmouss (1250m), Had bouhssoussen (forêt sidi çalah ,1450m), Moulay Bouaazza (1040m), Mbarkyene (820m);

- $\quad$ Silene diversiflora Otth.

Th - pâturages terreux, champs incultes et moissons; plaines et basses montagnes - SA SH - localité : Tout le Maroc.

\section{CAPPARACEAE}

\section{- Cleome L.}

- $\quad$ Cleome violaceae $L$.

$\mathrm{Th}$ - forêts claires et graviers des torrents, jusque vers $1500 \mathrm{~m}$ - SA SH - HA MA Mam Man (Monts de Zaine) R - localité : Monts d'Aguelmouss (jbel Torziane $(1630 \mathrm{~m})$;

\section{CISTACEAE}

\section{- Cistus L.}

\section{- $\quad$ Cistus salviifolius $L$.}

Ch (Nph) - Forêt et montagnes ne dépasses pas 2100m ; calcifuges - SA SH H - AA HA Man Mam Om LM R- localité : Aguelmouss (1250m), Had bouhssoussen (forêt sidi çalah ,1450m), Moulay Bouazza (1040m), Mbarkyene (820m) ; Al Go-Ida (1450m) ;

\section{- $\quad$ Cistus monspeliensis $L$.}

Nph - Foreet et matorrals ; basses et moyennes montagnes - SA SH - HA MA Mam Man Om LM R - localité : Aguelmouss (1250m), Had bouhssoussen (forêt sidi çalah ,1450m), Moulay Bouazza (1040m), Mbarkyene (820m); Al Go-Ida (1450m) ;

\section{- $\quad$ Cistus albidus $L$.}

$\mathrm{Nph}$ - Matorrals et foreet claires ; plaines et basses montagnes - SA SH H Mam Man Om LM R - localité : Aguelmouss (1250m), Had bouhssoussen (forêt sidi çalah ,1450m), Moulay Bouazza (1040m), Mbarkyene (820m); Al Go-Ida (1450m);

\section{- $\quad$ Tuberaria (Dunal) Spach.}

- $\quad$ Tuberaria echoides Lam. 


\section{= Helianthemum echoides Lam.}

Th - pâturages sablonneux en plaines - SA SH- Mam Man R - localité : Oulmès (1170m), Tiddass (330m), Maaziz (520m);

- $\quad$ Tuberaria guttata $L$.

= Helianthemum guttatum $\mathbf{L}$.

Th - Ermes, matorrals et clairières su sol sablonneux ne dépasses pas 1500m

- SA SH H - localité : Tidass (450m), Maaziz (520m), Oulmès (1170m) ;

- $\quad$ Helianthemum Miller.

- Helianthemum ledifolium Miller.

Th - Matorrals claires, ermes et steppes - Tout le Maroc - EMAT

- Helianthemum intermidium Pers.

Th - Ermes des plaines et des basses montagnes - A SA SH - localité : Tout le Maroc sauf Ms ;

- Helianthemum cinereum Pers.

$\mathrm{Ch}$ - foreet claires et matorrals sur des terrains rocailleux des basses et moyennes montagnes - SA SH H - localité : Tout le Maroc

CONVOLVULACEAE

- Convolvulus L.

- Convolvulus siculus $\mathbf{L}$.

Th - sols non sablonneux ; plaines, basse et moyennes montagnes - A SA SH H - localité : Tout le Maroc.

- Convolvulus arvensis $L$.

G - cultures, jachères, rocailles ; bords de la route ; plaines et montagnes SA SH H - localité : Tout le Maroc ;

- Convolvulus leucochnous Benoist

$\mathrm{H}$ - forêts dégradés, rocailles, ébulis calcaires, plaines et basses montagnes SA SH H - HA MA Mam Man R - localité : Aguelmouss (1250m), Had bouhssoussen (forêt sidi çalah ,1450m), Moulay Bouaazza (1040m), Mbarkyene (820m) ; Al Go-Ida (1450m) ; EM.

\section{CRASSULACEAE}

\section{- Crassula L.}

\section{- $\quad$ Crassula vaillantii Roth.}

Th - plaines et basses montagnes, SA SH H - AA HA MA Mam - localité : Aguelmouss (1250m), Had bouhssoussen (forêt sidi çalah ,1450m), Moulay Bouaazza (1040m);

\section{- Umbilicus DC}

- Umbilicus rupestris Dandy.

$\mathrm{G}$ - Rochers, vieux murs, sous bois des plaines et des montagnes ne dépasse pas 1800m - SA SH H - HA MA Mam Man Om LM R - localité : Aguelmouss (1250m), Had bouhssoussen (forêt sidi çalah ,1450m), Moulay Bouaazza (1040m), Mbarkyene (820m), Algo-Ida (1450m). 
- Umbilicus horizontalis (Guss) DC.

G - Rochers, vieux murs, sous bois; plaines et moyennes montagnes - SA SH H - Ms AA HA MA Mam Man Op Om LM R - localité : Aguelmouss (1250m) Had bouhssoussen (forêt sidi çalah ,1450m), Moulay Bouaazza (1040m), Mbarkyene (820m) ; Oulmès (1170m), Maaziz (520m) ;

\section{- $\quad$ Sedum L.}

- Sedum hirsutum ALL.

Ch - roches ombragés des plaines et des basses montagnes -SA SH H - EM.

\section{- $\quad$ Sedum baeticum Rouy.}

$\mathrm{Ch}$ - roches ombragés des plaines et des basses montagnes -SA SH H localité : Moulay bouazza (1040m), Maaziz (1170m), Algo-Ida (1450m) ;

\section{- $\quad$ Sedum rubens $L$.}

Th - rocailles, graviers, matorrals, pâturages pierreux des plaines et des basses montagnes - A SA SH H - AA HA MA Man Mam Op R - localité : Aguelmouss (1250m), Had bouhssoussen (forêt sidi çalah ,1450m), Moulay Bouaazza (1040m), Mbarkyene (820m).

\section{- $\quad$ Sedum modestum Ball.}

Th - rocailes, fissures des rochers, souches des arbres; plaines et basses montagnes - SA SH H - AA HA MA Mam Man -localité : Aguelmouss (1250m), Had bouhssoussen (forêt sidi çalah ,1450m), Moulay Bouaazza (1040m), Mbarkyene (820m) - EM.

\section{- Sedum album L.}

Ch - rochers, murs, rocailles et pâturages pierreux; plaines, basses et moyennes montagnes - A SA SH - HA MA Mam Man Op R - localité : Aguelmouss (1250m) , Had bouhssoussen ( forêt sidi çalah ,1450m), Moulay Bouaazza (1040m), Mbarkyene (820m) ; Al Go-Ida (1450m) ;

\section{- $\quad$ Sedum rupestre $L$.}

Ch - forêts, rochers et rocailles ombragés des montagnes - SH H - MA Man R - localité : Aguelmouss (1100m) et Had bouhssoussen (forêt sidi çalah ,1450m);

\section{- $\quad$ Sedum sediforme (Jacq) Pau.}

$\mathrm{Ch}$ - surfaces rocheuses ; plaines, basses et moyennes montagnes - A SA SH $\mathrm{H}$ - localité : Tout le Maroc rare dans Op et Ms.

\section{- $\quad$ Sedum jahandiezzii Batt.}

Th - dépressions des rochers siliceux, plaines et basses montagnes - SA SH H - MA Mam Man - Localité : Maaziz (520m) et Oulmés (1170m) - EM.

\section{CUPRESSACEAE}

\section{- $\quad$ Tetraclinis Masters}

- $\quad$ Tetraclinis articulata (Vahl) Masters (Thuya de bérbérie)

Ph - Plaine, basses et moyennes montagnes - SA SH - AA HA MA Man OP Om LM R - localité : Aguelmouss (1250m), Had bouhssoussen (forêt sidi 
çalah ,1450m), Moulay Bouaazza (1040m), Mbarkyene (820m) ; Al Go-Ida (1450m) ;

- Juniperus $L$.

- Juniperus oxycedrus

Ph(Nph)- Forêts et matorrals ; Plaines et montagnes - SA SH H- As AA HA MA Man Om Op LM R - localité : Aguelmouss (1250m) , Had bouhssoussen ( forêt sidi çalah ,1450m), Moulay Bouaazza (1040m), Mbarkyene (820m) , Al Go-Ida (1450m), Oulmès(1170m) ;

\section{CRUCIFEREAE}

- Alyssum L.

- $\quad$ Alyssum simplex Rudolphi.

Th - SH H - Pâturages, champs et clairières des forêts - EM - localité : Toutes les divisions géographiques du Maroc sauf Ms :

- Cardamine L.

- $\quad$ Cardamine hirsuta $L$.

Th - forêts et matorrals des plaines, basses et moyennes montagnes - SA SH H - AA HA MA Mam Man Om LM R - localité : Aguelmouss (1250m), Had bouhssoussen (forêt sidi çalah ,1450m), Moulay Bouazza (1040m), Mbarkyene (820m) ; Al Go-Ida (1450m) ;

- $\quad$ Erucastrum C. Presl

- $\quad$ Erucastrum laevigatum Maire.

$\mathrm{H}$ - plaines des basses montagnes - SA - MA Man R - localité : Aguelmouss (1250m), forêt sidi Hssein (1350m) ;

\section{- $\quad$ Sinapis L. (bahamou)}

- $\quad$ Sinapis arvensis $L$.

Th - ermes, cultures - plaines, basses et moyennes montagnes - A Sad Saf SH - HA MA Man Op Om LM R - localité : Oulmès (1170m), Tiddass (430m), Maaziz (520m) ;

\section{- $\quad$ Sinapis alba $\mathbf{L}$.}

Th - matorrals, ermes, cultures, rocailles et décombres ; plaines, basses et moyennes montagnes - A SA SH - AA HA MA Mam Man Op LM R localité : Toute la zone d'étude ;

\section{- $\quad$ Isatis $L$.}

\section{- $\quad$ Isatis odorata $L$.}

Th - plaines et basses montagnes - A SA SH H- HA MA Mam Man Op R localité : Toute la zone d'étude ;

- Sisymbrella spach.

- $\quad$ Sisymbrella boissieri Cosson.

H- sources et ruisseux des montagnes - SH H - HA MA Man Om R localité : Oulmès (1170m),

\section{- $\quad$ Sisymbrella officinale $L$.}


Th - plaines et moyennes montagnes - A SA SH -localité : Tout le Maroc sauf Ms et As ;

\section{- $\quad$ Sisymbrella erysimoides Desf.}

Th - plaines, basses et moyennes montagnes - A SA - localité : Tout le Maroc.

\section{CUCURBITACEAE}

- Bryonia L.

- $\quad$ Bryonia dioica Jacp . ( anbe eddibe)

G - Broussailles, haies, lieux ombragés ; plaines, basses et moyennes montagnes - A SA SH H - Tout le Maroc.

\section{ERICACEAE}

- $\quad$ Arbutus L.

\section{- $\quad$ Arbutus unedo $L$.}

Ph (Nph) - forêts et matorrals, plaines, basses et moyennes montagnes - SA SH H - AA HA MA Mam Man Om LM R - localité : Aguelmouss (Jbel Torziane, 1630m), Moulay Bouazza (1040m), Mbarkyene (820m) ; Al GoIda (1450m), Oulmès (1170m) ;

\section{EPHEDRACEAE}

\section{- $\quad$ Ephedra L.}

- $\quad$ Ephedra altissima (Ephédra élevé)

(Ph)(Nph) - SA SH - Forêts, broussailles, haies, rochers, plaines, basses et moyennes montagnes - localité : Tout le Maroc.

\section{EUPHORBIACEAE}

\section{- Euphorbia L.}

- $\quad$ Euphorbia squamigera Loisel.

$\mathrm{Ch}$ - milieux rocheux ; plaines, basses et moyennes montagnes - SA SH H AA HA Man Op Om LM R - localité : Oulmès (1170m) ;

\section{- $\quad$ Euphorbia pelpus $L$.}

Th - plaines, basses et moyennes montagnes - A SA SH H - localité : Tout le Maroc rare dans Ms et As ;

\section{- $\quad$ Euphorbia hirsuta L.}

= Euphorbia pubescens Vahl.

$\mathrm{H}$ - zones humides, bords de ruisseux; plaines, basses et moyennes montagnes - SA SH H - localité : Tout le Maroc ;

\section{- $\quad$ Euphorbia clementei Boiss.}

Ch - plaines, basses et moyennes montagnes - SA SH H - AA HA MA Mam Man Om LM R- localité : Toute la zone d’étude - EM.

\section{- $\quad$ Euphorbia rimarum Cosson \& Balansa.}

$\mathrm{Ch}(\mathrm{H})$ - fissures des rochers ; plaines, basses et moyennes montagnes - A SA -HA MA central Mam - localité : Aguelmouss (1250m), Had 
bouhssoussen (forêt sidi çalah ,1450m), Moulay Bouazza (1040m), Mbarkyene (820m) ; Al Go-Ida (1450m) - EM.

- $\quad$ Euphorbia medicaginea Boiss.

Th - plaines et basses montagnes - A SA SH H - MA Mam Man Om LM R - localité : Tout le Maroc sauf Ms ;

- $\quad$ Euphorbia fulcata $L$.

Th - ermes, cultures, jachères ; plaines, basses et moyennes montagnes - A SA SH H - localité : Tout le Maroc sauf Ms ;

- $\quad$ Euphorbia sulcata Loisel.

Th - plaines, basses et moyennes montagnes - A Sad Saf SH H - localité : Tout le Maroc.

- $\quad$ Mercurialis $L$.

- $\quad$ Mercurialis ambigna $L$.

Th - cultures, bords de chemins, rochers; plaines, basses et moyennes montagnes - SA H - localité : Tout le Maroc ;

- $\quad$ Ricinus $L$.

- $\quad$ Ricinus communis $L$.

$\mathrm{Ph}$ - bords de routes, haies, décombres ; plaines et basses montagnes - A SA SH - localité : Tout le Maroc.

FABACEAE (LEGUMINEUSES)

Anagyris $L$.

- $\quad$ Anagyris foetida $L$.

Nph - plaines et basses montagnes - A SA SH H - AA HA MA Mam Man LM R- Toutes les divisions sauf Ms.

- $\quad$ Argyrolobium Ecklon \& Zeyher

- $\quad$ Argyrolobium zanonii L.

= Genista argentea $\mathbf{L}$.

$\mathrm{Ch}$ - forêtS claires, matorrals, ermes, rocailles, plaines et basses montagnes SA SH H - Toutes les divisions sauf Ms.

- $\quad$ Anthyllis L.

- $\quad$ Anthyllis vulneraria $L$.

$\mathrm{H}$ (Th) - forêts, matorrals, ermes et steppes ; plaines et montagnes - A SA SH H - Maroc non saharien - EM.

- $\quad$ Anthyllis cornicina $L$.

Th - forêts claires des basses montagnes siliceux - SA SH - aa ma Man (zaine) R - localité : Moulay Bouazza (1040m), Mbarkyene (820m) ; Al GoIda (1450m), Oulmès (1170m), Maaziz (520m), Tiddass (430m).

- $\quad$ Astragalus L.

- $\quad$ Astragalus caprinus L.

$\mathrm{H}$ - clairiéres des forêts, steppes, rocailles ; plaines, basses et moyennes montagnes - SA- Tout le Maroc ; 


\section{- $\quad$ Astragalus glaux $L$.}

$\mathrm{H}$ - clairiéres des forêts, pelouses sèches ; plaines, basses et moyennes montagnes -A SA SH H - AA HA MA Man Mam Om LM R- large distribution au niveau de la zone d'étude ;

- $\quad$ Astragalus cymbicarpos Brot.

Th - basses montagnes siliceux - SH H - MA Man - localité : Aguelmouss (1250m), Had bouhssoussen (forêt sidi çalah ,1450m), Moulay Bouazza (1040m), Mbarkyene (820m) ; Al Go-Ida (1450m), Oulmès (1170m), Maaziz (520m), Tiddass $(430 \mathrm{~m})$;

\section{- $\quad$ Ceratonia L.}

\section{- $\quad$ Ceratonia siliqua $L$.}

$\mathrm{Ph}$ - plaines et basses montagnes ; spontané et cultivé - SA SH- As AA HA MA Mam Man Om LM R - localité : Aguelmouss (1250m), Had bouhssoussen ( forêt sidi çalah ,1450m), Moulay Bouazza (1040m), Mbarkyene (820m) ; Al Go-Ida (1450m), Oulmès (1170m) ;

- Coronilla $L$.

- $\quad$ Coronilla scorpioides L. (rbiaa el aagreb)

Th - cultures, moissons, jachères, rocailles ; plaines et basses montagnes SA SH - Tout le Maroc.

\section{- $\quad$ Coronilla valentina $L$.}

Nph - forêts claires, matorrals, rochers calcaires ; surtout ombragés ; plaines, basses et moyennes montagnes - A SA SH H - AA HA MA Mam Man Om LM R.

\section{- $\quad$ Cytisus $L$.}

- $\quad$ Cytisus arboreus (Desf) DC.

Nph - Forêts, matorrals, rocailles, plaines basses et moyennes montagnes SA SH H - AA HA MA Mam Man Om LM R - localité : Toute la zone d'étude;

- Cytisus granadiflorus (Brot) DC.

$\mathrm{Nph}$ - forêts et matorrals des basses et moyennes montagnes siliceuses - SA SH H - HA MA Man LM R - localité : Aguelmouss (1250m), Had bouhssoussen ( forêt sidi çalah ,1450m), Moulay Bouazza (1040m), Mbarkyene (820m) ; Al Go-Ida (1450m), Oulmès (1170m), Maaziz (520m), Tiddass(430m) - EM ;

\section{- $\quad$ Genista L.}

- $\quad$ Genista scorpius $L$.

Nph - forêts et matorrals des basses et moyennes montagnes siliceuses - SA SH H - HA MA Man LM R - localité : Aguelmouss (1250m), Had bouhssoussen (forêt sidi çalah ,1450m), Moulay Bouazza (1040m), Mbarkyene (820m) ; Al Go-Ida (1450m), Oulmès (1170m), Maaziz (520m), Tiddass (430m) - EM ; 
- $\quad$ Genista quadriflora Munby.

Nph (Ch) - montagnes non calcaires - SA SH H - HA MA Man LM R large distribution au niveau de la zone d'étude ;

\section{- $\quad$ Genista anglica $L$.}

Nph (Ch) - marais, bords de ruisseaux, fonds humides de ravins ; plaines siliceuses - SH H - Man - localité : Nph - forêts et matorrals des basses et moyennes montagnes siliceuses - SA SH H - HA MA Man LM R localité : Oulmès (1170m), Maaziz (520m), Tiddass (430m) ;

\section{- Genista hirsuta Vahl.}

Ch (Nph) - forêts claires, matorrals, rocailles ; plaines et basses montagnes A Sad SH - Man Op Om LM R -localité : Nph - forêts et matorrals des basses et moyennes montagnes siliceuses - Sad SH H - HA MA Man LM R - localité : Oulmès (1170m), Maaziz (520m), Tiddass (430m) - EM ;

- $\quad$ Lathyrus L.

- $\quad$ Lathyrus setifolius $L$.

Th - plaines et basses montagnes - A SA SH H - HA MA Mam Man R localité : Oulmès $(1170 \mathrm{~m})$;

- $\quad$ Lathyrus cicerea $L$.

Th - champs, matorrals, ermes ; plaines et basses montagnes - A SA SH H HA MA Mam Man Op Om LM R - Localité : Toute la zone d’étude ;

\section{- $\quad$ Lotus L.}

- $\quad$ Lotus weilleri Maire.

Th - forêts et matorrals des basses montagnes siliceux - SA SH - Man localité : Jbel El harcha (930m) - EM ;

- Lotus marrocanus Ball.

$\mathrm{H}$ - clairières, matorrals, ermes ; plaines, basses et moyennes montagnes - A SA SH - AA HA Mam Man - EM ;

- Medicago L.

- $\quad$ Medicago polycerabtia $L$.

Th - clairières et pâturages rocailleux; plaines, basses et moyennes montagnes - A SA SH H - Tout le Maroc ;

- $\quad$ Medicago lupulina $L$.

H (Th) - ermes, lieux humides, sables fluviatiles; plaines basses et moyennes montagnes - A SA SH H - AA HA MA Mam Man Om LM R ;

- $\quad$ Medicago minima $L$.

Th - forêts, matorrals, ermes, champs ; basses montagnes - A SA SH H Tout le Maroc.

\section{- Retam Rafin.}

- $\quad$ Retama monosperma L.

Nph - matorrals et ermes pierreux ; plaines et basses montagnes - SA SH Ms AA HA MA Mam Man Om LM R - localité : Toute la zone d’étude ; 
- $\quad$ Pisum L.

- $\quad$ Pisum calaticus Ascherson \& Graebne.

Th - forêts claires, matorrals, eboulis ; plaines et basses montagnes - A SA SH H - HA MA Man Mam Om LM R ;

\section{- $\quad$ Scorpiurus L.}

- $\quad$ Scorpiurus muricatus L. (oudinate naaja)

Th - ermes et champs des plaines et basses montagnes - A SASH - AA HA MA Mam Man Op Om LM R ;

- Trifolium $L$.

- $\quad$ Trifolium compestre Schreber.

Th - champs sablonneux ; plaines, basses et moyennes montagnes - A SA SH H - AA HA MA Mam Man Op Om LM R ;

- $\quad$ Trifolium filiforme $\mathrm{L}$.

Th - SH H - Marais et pelouses humides - MA Mam Man LM R ;

- Trifolium fragiferum $\mathbf{L}$.

$\mathrm{H}$ - Prairies humides, sables des rivières, plaines, basses et moyennes montagnes - A SA SH H - HA MA Mam Man Om LM R ;

- Trifolium spermosum $\mathbf{L}$.

Th - lieux herbeux humides des plaines - SA SH - Mam Man Om LM R ;

- $\quad$ Trifolium glomeratum $\mathbf{L}$.

Th - forêts, matorrals, ermes ; plaines, basses et moyennes montagnes - A SA SH H - AA HA MA Mam Man Om LM R ;

- $\quad$ Trifolium suffocatum $\mathbf{L}$.

Th - clarières des forêts, pâturages - plaines, basses et moyennes montagnes - Sad SH H - Man R - localité : Oulmès (1170m), Maaziz (520m), Tiddass (430m) ;

\section{- $\quad$ Trifolium repens $L$.}

$\mathrm{H}$ - prairies humides, bords des eaux; plaines, basses et moyennes montagnes - A SA SH H - HA MA Mam Man Om LM R ;

- Trifolium hirtum All.

Th - forêts, matorrals, ermes ; basses et moyennes montagnes - SH H - MA Man Om R - localité : Aguelmouss (1250m), Had bouhssoussen (forêt sidi çalah ,1450m), Moulay Bouazza (1040m), Mbarkyene (820m); Al Go-Ida (1450m), Oulmès (1170m), Maaziz (520m), Tiddass $(430 \mathrm{~m})$;

- $\quad$ Trifolium angustifolium $\mathbf{L}$.

Th - matorrals , ermes, pâturages sablonneux et rocailles , plaines , basses et moyennes montagnes- A SA SH H - AA HA MA Mam Man Om LM R ;

- Trifolium sellatum $L$.

Th - clairières des forêts , matorrals, ermes, champs, plaines , basses et moyennes montagnes - SA SH H - AA HA MA Mam Man Om LM R ;

\section{- $\quad$ Trifolium gemellum Willd.}


Th - clairières des forêts , matorrals , ermes, champs , plaines , basses et moyennes montagnes , SA SH H - AA HA MA Mam Man Om LM R localité : $\quad$ Aguelmouss(1250m), Al go-aida(1475m) ,Had bouhssoussen(1450m);

- Ononis L.

- $\quad$ Ononis breviflora DC.

Th - forêts claires, matorrals, ermes et champs argileux des plaines - SA Mam Man LM - localité : Oulmès (1170m), Maaziz (520m) ;

- Ononis hispanica $L$.

$\mathrm{Ch}$ - forêts claires, matorrals, ermes ; plaines, basses et moyennes montagnes - SA SH - Toutes les divisions de Maroc ;

- $\quad$ Ononis spinosa $L$.

Ch - matorrals, champs, pâturages ; plaines, basses et moyennes montagnes - A SA SH H - AA HA MA Mam Man Om LM R.

- Ornithopus L.

- Ornithopus compressus $L$.

Th - clairières des forêts, pâturages des terrains sablonneux; plaines et basses montagnes - A SA SH H - AA HA MA Mam Man R ;

- $\quad$ Vicia L.

- Vicia narbonensis $L$.

Th - forêt claires, matorrals et ermes des plaines - A SA SH - AA MA Mam Man Op Om LM R ;

- Vicia lutea $L$.

Th - cultures, clairières, ermes ; plaines, basses et moyennes montagnes - A SA SH H - Tout le Maroc ;

- Vicia amphicarpa $L$.

Th - forêts claires, matorrals, ermes ; plaines, basses et moyennes montagnes - A SA SH H - AA HA MA Mam Man Op Om R - localité : Aguelmouss (1250m), Had bouhssoussen (forêt sidi çalah ,1450m), Moulay Bouazza (1040m), Mbarkyene (820m) ; Al Go-Ida (1450m);

\section{- Vicia angustifolia $L$.}

Th -forêts claires, matorrals, ermes ; plaines, basses et moyennes montagnes - SA SH H- Ms As AA MA Mam Man LM R - large distribution au niveau de la zone d'étude ;

\section{FAGACEAE}

\section{- Quercus L. \\ - Quercus suber $L$.}

$\mathrm{Ph}$ - plaines, basses et moyennes montagnes, sur sols siliceux - SA SH HA MA Man Om LM R- localité : Aguelmouss (1250m), Had bouhssoussen (forêt sidi çalah ,1450m), Moulay Bouazza (1040m), Mbarkyene (820m); Al Go-Ida (1450m), Oulmès (1170m), Maaziz (520m), Tiddass (430m) ; 
- $\quad$ Quercus ilex

= Quercus rotundifolia Lam.

$\mathrm{Ph}$ - basses, moyennes et hautes montagnes, ne dépasses pas 2900m - SA SH $\mathrm{H}$ - localité : Tout le Maroc sauf Ms et Op ;

\section{GERANICEAE}

- $\quad$ Erodium L'Hèr \& Aiton

- $\quad$ Erodium guttatum (Desf.) Willd

$\mathrm{H}$ - steppes, pâturages rocailleux et terreux ; plaines, basses et moyennes montagnes - SA SH - localité : Toutes les divisions du pays ;

- $\quad$ Erodium sebaceum Delile.

$\mathrm{H}$ - pâturages terreux des basses et moyennes montagnes - SH H - MA Man - localité : Oulmès (1170m) et Aguelmouss (1250m) - EM ;

\section{- $\quad$ Erodium mouretii Pitard.}

$\mathrm{H}$ - fentes des roches quartzites ; calcifuge - SA SH - MA Mam Man localité : Oulmès (1170m), Moulay bouazza (1040m) ;

- $\quad$ Erodium brachycarpum (Godron.) Thell.

Th - sols squelettiques sur grès rouges en général ; basses montagnes - SA SH - HA Mam Man R - localité : Tout le plateau central ;

\section{- $\quad$ Erodium malacoides (L.) L'Hèr}

Th - clairières des forêts, pâturages, champs ; plaines, basses et moyennes montagnes - A SA SH H - Ms AA HA MA Mam Man Op LM R - localité : Toute la zone d'étude - EMA ;

\section{- $\quad$ Erodium chium (L.) Willd}

Th - SA SH H - localité : Toutes les divisions du Maroc - EM ;

\section{Geranium lucidum $\mathbf{L}$.}

Th - Forêts, matorrals, rochers et rocailles frais et ombragés, plaines, basses et moyennes montagnes - SA SH H ; HA MA Man Om LM R - localité : Aguelmouss (1250m), Had bouhssoussen (forêt sidi çalah ,1450m), Moulay Bouazza (1040m), Mbarkyene (820m); Al Go-Ida (1450m), Oulmès (1170m), Maaziz (520m), Tiddass (430m);

\section{- $\quad$ Geranium rotundifolium $L$.}

Th - Forêts claires, rocailles ombragées, plaines, basses et moyennes montagnes ; SA SH H - localité : Toutes les divisions sauf Ms.

JUNCACEAE

\section{- Juncus maritimus Lam.}

$\mathrm{H}$ - Lieux humides, bords de rivières et lacs, plaines, basses et moyennes montagnes Ne dépasse pas 1400m - SA SH H - HA MA Mam Man LM R localité : dans toutes la région d’étude au niveau des zone humides ;

- Juncus articulatus $L$.

G - Lieux humides - H - HA MA Mam Man LM R- localité : Oulmès (1170m), tiddass (430m), Moulau bouazza (1040m) ; 
- Juncus bufonius $L$.

G - Lieux humides - H - Ms As AA HA MA Mam Man Om LM R localité : Toute la région d'étude au niveau des lieux humides - RR - EM ;

- Juncus effuses $L$.

(H) - Lieux humides - H - MA Man R - localité : Toute la zone d’étude ;

\section{LAMIACEAE}

- Ballota L.

- Ballota hirsuta Bentham.

Ch - matorrals, rochers ; plaines et basses montagnes - A SA SH H localité : Maroc non saharien - EMA ;

- Lavandula L.

- $\quad$ Lavandula multifida $L$.

Ch - plaines et basses montagnes - A SA SH -localité : Maroc non saharien ;

- $\quad$ Lavandula dentata $L$.

Ch (Nph) - plaines et basses montagnes - A SA SH - localité : Maroc non saharien ;

- $\quad$ Lavandula stoechas $L$.

Ch (Nph) - plaines et basses montagnes - A SA SH -localité : Maroc non saharien - EM ;

- Lavandula pedunculata (Miller) Cav.

Ch (Nph) - plaines et basses montagnes - A SA SH - localité : Maroc non saharien ;

- $\quad$ Marrubium L.

- $\quad$ Marrubium vulgare $L$.

$\mathrm{Ch}$ - plaines, basses et moyennes montagnes - A SA SH H - Tout le Maroc ;

- Marrubium echinatum Ball.

$\mathrm{H}$ - forêts et matorrals des basses et moyennes montagnes - SH H - HA MA Man R - localité : Aguelmouss (1250m) et Had Bouhssoussen 1450m) $\mathrm{EM}$;

- $\quad$ Mentha L.

- $\quad$ Mentha pulgium $L$.

$\mathrm{H}$ - lieux humides des plaines, basses et moyennes montagnes - SA SH H Maroc non saharien ;

- $\quad$ Mentha longifolia (L.) Hudson.

$\mathrm{H}$ - bords de ruisseux, et des dayas, lieux humides; basses et moyennes montagnes - SH SH - HA MA Mam Man Op - localité : Toute la zone d'étude ;

\section{- $\quad$ Mentha rotundifolia $L$.}


$\mathrm{H}$ (th) - bords des eaux ; plaines, basses et moyennes montagnes - SA SH H

- Tout le Maroc;

- $\quad$ Thymus L.

- $\quad$ Thymus zygis $L$.

Ch - clairières des forêts, pâturages pierreux des basses et moyennes montagnes - SA SH H - HA MA Mam Man - localité : Aguelmouss (1250m), Had bouhssoussen (forêt sidi çalah ,1450m), Moulay Bouazza (1040m), Mbarkyene (820m) ; Al Go-Ida (1450m), Oulmès (1170m) ;

\section{- $\quad$ Teucrium L.}

- $\quad$ Teucrium spinosum $L$.

Th - champs incultes, terrains argileux - SA - Mam Man R - localité : Oulmès (1170m), Maaziz (520m), Tiddass (430m) ;

\section{- $\quad$ Teucrieum decipiens Cosson \& Balansa}

Th - clairières des forêts, rocailles ; plaines et basses montagnes - SA SH HA MA Mam Man Om LM R -localité : Toute la zone d’étude - EM ;

- $\quad$ Teucrieum zaianum Emberger \& Maire.

$\mathrm{Ch}$ - rochers quartzitiques et forêts rocailleuses des basses montagnes - SH Man- localité : Oulmès (1170m), Maaziz (520m), Tiddass (430m) - EM ;

\section{- Stachys L.}

\section{- Stachys arenaria Vahl.}

Ch - forêts clairs, matorrals , ermes , steppes ; plaines, basses et moyennes montagnes - SA SH - AA HA MA Mam Man R - localité : Aguelmouss (1250m) , Had bouhssoussen ( forêt sidi çalah ,1450m) , Moulay Bouazza $(1040 \mathrm{~m})$, Mbarkyene (820m); Al Go-Ida (1450m), Oulmès (1170m), Maaziz (520m), Tiddass(430m) - EM ;

- $\quad$ Stachys circinata L'Hèr.

$\mathrm{H}$ - Forêts, pâturages et broussailles des basses montagnes - SH H - MA Man LM R - localité : El harcha (930m) - EM - RR ;

\section{- $\quad$ Stachys zaiana Emberger \& Maire.}

$\mathrm{H}$ - Forêts, pâturages et broussailles des basses montagnes - SH H -Man localité : Oulmès (1170m), Maaziz (520m), Tiddass (430m) - EM ;

\section{- $\quad$ Stachys arvensis $L$.}

Th - forêts et matorrals claires, lieux incultes, champs ; plaines et basses montagnes - SA SH H - Maroc non saharien ;

\section{- Origanum L.}

\section{- $\quad$ Origanum compactum Bentham}

Ch - forêts, matorrals, ermes, rocailleux, rochers; plaines et basses montagnes - A SA SH - MA Mam Man R - Toute la zone d’étude ;

\section{LAUREACEAE}

\section{- $\quad$ Laurus L.}

- $\quad$ Laurus nobilis L. (chajarate sidna moussa) 
Ph - Ravins et roches des forêts et matorrals ; plaines et montagnes qui ne dépasse pas 1800m - SH H - MA Man - localité : Oulmès (1170m), Maaziz (520m);

\section{LILIACEAE}

- $\quad$ Asparagus albus L. (Asperge blanche)

Ph - Broussailles et forêt des montagnes - SH H - AS HA MA Mam Man Op LM R- large distribution dans la zone d'étude.

- $\quad$ Asparagus stipularis Forsk.

Ph - Forêts claires, les broussailles, les pâturages argileux et pierreux, des steppes des plaines et des montagnes - SH H - AS HA MA Mam Man Op LM R - large distribution dans la zone d'étude.

- $\quad$ Asphodelus acaulis Desf. (Asphodèle acaule)

G - Pâturages rocailleux, les clairières des forêts, des montagnes calcaires-

SH H - AS HA MA Mam Man Op LM R- large distribution dans la zone d'étude.

- $\quad$ Asphodelus microcarpus Salzm. et Viv. (Asphodèle)

G - SA SH - Pâturages rocailleux et les clairières des forêts - AS HA MA Mam Man Op LM R - large distribution dans la zone d'étude.

- $\quad$ Muscari Comosum (L.) Miller.

G - champs, les lieux incultes sablonneux ou rocailleux de la plaine et les clairières des forêts des montagnes - SA SH ; AS HA MA Mam Man Op LM R- large distribution dans la zone d'étude.

- $\quad$ Urginia maritima L. (Scille de mer)

G - steppes, les pâturages, les broussailles et les forêts de la plaine et des basses montagnes - SA SH - AS HA MA Mam Man Op LM R - large distribution dans la zone d'étude.

\section{MALVACEAE}

\section{Lavatera $L$.}

- Lavatera trimestris $L$.

Th - Champs, plaines et basses montagnes - A SA SH H - MA Man Op Om LM R ;

\section{- $\quad$ Lavetera maroccana Maire.}

Th - Dayas, champs, plaines - A SA -Mam Man Op - localité: Oulmès (1170m);

- $\quad$ Lavetera olbia L. (khobbiza)

Nph - Ravius et ruisseaux ; plaines et basses montagnes - SA SH H - MA LM R - large distribution dans la zone d'étude ;

- Lavetera cretica $L$.

Th - lieux incultes, décombres ; plaines et basses montagnes - A SA SH H AA HA MA Mam Man Om LM Op ;

\section{Malva $L$.}


- Malva hispanica L.

Th - Moissons, jachères, clairières ; plaines, basses et moyennes montagnes - A SA SH H - HA MA Mam Man LM Om R ;

- Malva parviflora $L$.

Th - chemins, décombres, champs incultes ; plaines et basses montagnes SA SH - Tout le Maroc ;

\section{- $\quad$ Malva sylvestris $L$.}

$\mathrm{H}$ - Champs et lieux incultes ; décombres ; plaines et montagnes - Tout les bioclimats - AA HA MA Mam Man Om Op LM R - Tout le Maroc sauf Ms ;

\section{MORACEAE}

\section{- $\quad$ Ficus L.}

- $\quad$ Ficus carica L. (Figuier)

$\mathrm{Ph}$ - plaines, basses et moyennes montagnes - A SA SH H - Tout le Maroc sauf Ms ;

\section{PAPAVERACEAE}

\section{- Fumaria L.}

- $\quad$ Fumaria parviflora Lam.

Th - champs, lieux incultes - SA SH - Tout le Maroc.

- $\quad$ Fumaria densiflora DC.

Th - Champs, lieux incultes, cultures - A SA SH - Tout le Maroc.

- $\quad$ Fumaria officilnalis $L$.

Th - Champs, lieux incultes, bords de chemins, plaines, basses et moyennes montagnes - A SA SH H - Tout le Maroc, sauf Ms et As ;

- $\quad$ Fumaria rupestris Boiss \& Reuter.

Th - Rochers calcaires, schisteux et granitiques, matorrals; basses et moyennes montagnes - SA SH H - AA HA MA Mam Man Op Om LM R EM ;

\section{- $\quad$ Fumaria pulchra Lidèn.}

Th - Falaises calcaires - SA SH H - MA Mam Op - localité : Aguelmouss (1250m) ; Al Go-Ida 1475m) ;

\section{- $\quad$ Papaver $L$.}

- $\quad$ Papaver argemone $L$.

Th - Moissons, champs sablonneux des plaines et des basses montagnes SA SH - Man R - localité : Oulmès (1170m), Maaziz (430m) et Tiddass (480m) ;

\section{- $\quad$ Papaver hybridium $L$.}

Th -champs, lieux incultes, plaines, basses et moyennes montagnes - SA $\mathrm{SH}$ - Tout le Maroc ;

- $\quad$ Papaver somniferum L. (kharkhacha, afyum) 
Th -champs, lieux incultes, plaines, basses et moyennes montagnes - SA SH

- Tout le Maroc ;

- $\quad$ Papaver setigerum DC.

Th - Champs, moissons, rochers et rocailles des plaines et des basses montagnes - A SA SH - AA HA MA Mam Man Om LM R ;

- $\quad$ Papaver pinnatifidum Moris.

Th- Champs, plaines et basses montagnes - SA - MS AA HA Mam Man Op $\mathrm{R}$;

- Papaver dubium L.

Th - Cultures, steppes, matorrals et pâturages rocailleux des plaines et des montagnes- SA SH H - Tout le Maroc sauf le Ms.

PALMACEAE

- Chamaerops humilis L. (Palmier nain)

Th - SA SH ; AA HA MA Man Om R - localité : localité : Aguelmouss (1250m), Had bouhssoussen (forêt sidi çalah ,1450m), Moulay Bouazza (1040m), Mbarkyene (820m) ; Al Go-Ida (1450m), Oulmès (1170m), Maaziz (520m), Tiddass (430m), jusqu’au 1200m .

\section{PLANTAGINACEAE}

- $\quad$ Plantago L.

- $\quad$ Plantago afra L. = Plantago psyllium $\mathbf{L}$.

Th - milieux dégradés, lieux incultes, champs ; plaines et basses montagnes

- SA SH H - localité : Tout le Maroc ;

- $\quad$ Plantago major L.

Th - lieux humides des plaines, basses et moyennes montagnes - S A SH H - AA MA Mam Man 0p Om LM R ;

- $\quad$ Plantago logopus $L$.

Th - clairières des forêts, matorrals, ermes, champs, graviers des rivières ; plaines, basses et moyennes montagnes - SA SH H - Tout le Maroc

- $\quad$ Plantago lanceolata $\mathbf{L}$.

$\mathrm{H}$ - lieux humides ; plaines et moyennes montagnes - A SA Mam Man R

- $\quad$ Plantago bellardii All.

Th - plaines et basses montagnes - SA SH - Tout Le Maroc sauf Ms et As ;

- $\quad$ Plantago albicans $L$.

$\mathrm{H}$ - matorrals et ermes secs, steppes; plaines et basses montagnes - SA Tout le Maroc ;

- $\quad$ Plantago coronopus $\mathbf{L}$.

Th $(\mathrm{H})$ - forêts et matorrals dégradés, ermes, steppes ; milieux humides ; plaines, basses et moyennes montagnes - A SA SH - Tout le Maroc ;

\section{PRIMULACEAE}

- Anagallis L.

- $\quad$ Anagallis crassifolia Thore. 
H - lieux humides ruissellets des plaines - SH H - Man - localité : Oulmès (1170m), Maaziz (520m), Tiddass (430m);

- $\quad$ Anagallis monelli $L$.

$\mathrm{Ch}$ - Matorrals et rocailles ; plaines, basses et moyennes montagnes - A SA SH H - localité : Tout le Maroc ;

- $\quad$ Anagallis arvensis $L$.

Th - champs, rocailles, et clairières des forêts ; plaines basses et moyennes montagnes - A SA SH H - localité : Tout le Maroc ;

POLYGONACEAE

- $\quad$ Emex Campd.

- $\quad$ Emex spinosa L.

Th - Sols sableux, bords des chemins, SA SH H - Tout le Maroc ;

\section{Rumex $L$.}

- $\quad$ Rumex acetosella L.

$\mathrm{H}$ - lieux sablonneux et rocailles siliceuses ; plaines et basses montagnes SA SH H - HA MA Man Om LM R.

- $\quad$ Rumex thyrsoides Desf.

$\mathrm{H}$ - pâturages pierreux et rocailles jusqu'à 1800m - SASH H - AA HA MA Mam Mam Om LM R ;

- $\quad$ Rumex pulcher.

$\mathrm{H}$ - lieux humides, dayas et merjas - H - HA MA Mam Man Op LM R

- $\quad$ Rumex conglomeratus Murray.

$\mathrm{H}$ - Marais, dayas, lieux humides ; plaines et basses montagnes - A SA SH H - HA MA Mam Man Op Om LM R ;

\section{- Polygonum L.}

- Polygonum bellardii ALL.

Th - champs et cultures irriguées des plaines et des montagnes - SA SH HHA MA Mam Man R ;

- Polygonum aviculare $L$.

Th - champs lieux incultes, décombres, bords de chemin; plaines et des montagnes - SA SH H - Tout le Maroc - EM ;

POACEAE (Graminées)

- Bromus squarrosus L. (Brome)

Th - SA SH - pâturages et forêts de basses montagnes - Ms As AA HA MA Om R- Large distribution géographique.

\section{- $\quad$ Bromus rubens $L$.}

Th - SA SH - pâturages et forêt de basses montagnes -MS As AA HA MA Man Op Om LM R- Large répartition géographique ;

\section{- $\quad$ Bromus maditensis $L$.}

Th - SA SH - pâturages et forêt de basses montagnes- Ms As AA HA MA Man Op Om LM R - Large répartition géographique ; 
- Bromus lanceolatus Roth.

Th - SA SH - broussailles, cultures et pâturages - HA MA Mam Man Om LM R - Large répartition géographique ;

- $\quad$ Bromus sterilis $L$.

Th - SH H - forêt et pâturages - HA MA Mam R - Large répartition géographique.

- $\quad$ Dactylis glomerata L. (Dactyle glomérulé)

Th - SH H - Broussailles, pâturages et forêt des plaines et des montagnes As AA HA Mam Man Op Om LM R.

\section{- Hordeum murinum $L$.}

Th - SH H - Pâturages des plaines et des montagnes - toutes les divisions géographiques du Maroc - Large distribution géographique dans la zone d'étude et ne dépasse pas 1400m ;

- $\quad$ Lagurus ovatus L. (Queue de lièvre)

Th - SH H - Pâturages, forêt et broussailles - HA MA Man Op 0m LM R large distribution géographique dans la zone d'étude ;

- $\quad$ Lamarckia aurea $L$.

Th - SH H - Pâturages, plaines et des montagnes - Ms As AA HA MA Man Op 0m LM R- large distribution géographique dans la zone d'étude ;

- $\quad$ Melica ciliata $\mathbf{L}$.

Th - SH H - Pâturages, broussailles et clairières - AA HA MA Man Op 0m LM R - large distribution géographique dans la zone d’étude ;

- Melica cupani Guss.

Th - SH H - Pâturages et forêts des montagnes - AA HA MA Man Op 0m LM R - large distribution géographique dans la zone d'étude ;

- $\quad$ Lygeum spartum L. (Sparte), (gousmir)

Th - SH H - Pâturages, plaines et des montagnes - AA HA MA Man Op 0m LM R - large distribution géographique dans la zone d'étude ;

- Poa annua $L$.

Th - SH H - Pâturages, plaines et des montagnes - AA HA MA Man Op 0m LM R - large distribution géographique dans la zone d’étude ;

- Poa bulbosa L.

Th - SH H - Pâturages, plaines et des montagnes - AA HA MA Man Op 0m LM R - large distribution géographique dans la zone d'étude ;

- $\quad$ Phragmites communis Trin. (Roseau des marais)

Th - SH H - Bords des cours d'eaux - AA HA MA Man Op 0m LM Rlarge distribution géographique dans la zone d'étude ;

RANUNCULACEAE

- Adonis L.

- $\quad$ Adonis annua $L$. 
Th- champs - SA SH - Mam Man LM R - localité : Mbarkyene (820m) ; Al Go-Ida (1450m), Oulmès (1170m), Maaziz (520m), Tiddass(430m) ;

- $\quad$ Adonis microcarpus DC.

Th - steppes, champs cultivés - A SA - tout le Maroc ;

- Adonis aestivalis $L$.

Th - champs incultes, moissons, steppes - SA SH - Tout le Maroc ;

- $\quad$ Nigelle L.

- $\quad$ Nigelle damascene L.

Th - Champs et pâturages des plaines et des basses montagnes - A SA SH HA MA Mam Man LM R ;

\section{- $\quad$ Nigelle arvensis $L$.}

Th- champs et pâturages rocailleux; plaines et montagnes jusque vers 1800m - A SA SH H - HA MA Mam Man LM R ;

\section{- $\quad$ Ranunculus ficaria $L$.}

$\mathrm{H}$ - Prairies humides, forêts fraiches des plaines et des montagnes - SA SH H - HA MA Man R .

- $\quad$ Ranunculus falcatus $\mathbf{L}$.

Th - Steppes, champs, lieux sablonneux; plaines, basses et moyennes montagnes - SA SH H - AA HA MA Mam Man Om Op ;

- $\quad$ Ranunculus parviflorus $L$.

Th - Ravins humides et ombragés des plaines et basses montagnes- SA SH H - HA MA Man R ;

\section{- $\quad$ Ranunculus Lateriflorus DC.}

Th - Petites temporaires des basses et moyennes montagnes - SA SH - HA MA Mam R - localité : Aguelmouss (1250m), Had bouhssoussen ( forêt sidi çalah ,1450m) , Moulay Bouazza (1040m) , Mbarkyene (820m) ; Al Go-Ida (1450m);

\section{- $\quad$ Ranunculus bullatus L.}

$\mathrm{H}$ - clairières des forêts, ermes sablonneux et argileux, plaines et basses montagnes - SA SH H - AA MA Mam Man Om LM R ;

\section{- $\quad$ Ranunculus peltatus schrank.}

H- Eaux doux et saumâtres ou courantes ; plaines, basses et montagnes - SA $\mathrm{SH}$ - MA Mam Man Op R ;

\section{- $\quad$ Ranunculus scleratus.}

Th - Marais des ruisseaux - SA SH H - MA Man - localité : Oulmès (1170m), Maaziz (520m), Tiddass (430m);

- $\quad$ Ranunculus arvensis $L$.

Th- champs des plaines et des basses montagnes -SA SH H - AA HA MA Mam Man Op Om LM R ;

\section{- $\quad$ Ranunculus muricatus L.}


Th - lieux humides, bords des ruisseaux ; plaines et basses montagnes - A SA SH H - HA MA Mam Man Op Om LM R ;

\section{- $\quad$ Ranunculus paludosus Poiret.}

H- clairières des forêts, ermes des plaines et des montagnes - SA SH H - AA HA MA Mam Man Op Om LM R ;

- $\quad$ Ranunculus millefolius banks et Solander.

H- ermes rocailleux des moyennes montagnes - MA Man - localité : Maaziz (520m), Tiddass (430m) et ne dépasse pas 900m ;

- $\quad$ Ranunculus macrophyllus Desf.

$\mathrm{H}$ - bords des eaux, prairies marécageuses des plaines et des basses montagnes - A SA SH H - Mam Man Op Om LM R ;

\section{RESEDACEAE}

\section{- $\quad$ Reseda L.}

- $\quad$ Reseda alba L.

Th - pâturages rocailleux, argileux et sablonneux, rochers, vieux murs - S SA SH - Tout le Maroc - EM ;

\section{- $\quad$ Reseda lutea $L$.}

Th - pâturages pierreux et sablonneux des plaines et des basses montagnes SA SH H - Tout le Maroc - EMAT ;

\section{- $\quad$ Reseda phyterma $L$.}

Th - jachères, pâturages rocailleux ; plaines et basses montagnes - SA SH H - Tout le Maroc.

\section{RHAMNACEAE}

- $\quad$ Rhamnus L.

- $\quad$ Rhamnus lycioides $L$.

Nph - plaines et montagnes - Tout le Maroc - EMA ;

- $\quad$ Rhamnus alaternus $L$.

Nph - plaines et montagnes - SA SH H - AA HA MA Mam Man Om LM $\mathrm{R}$.

\section{Ziziphus Miller.}

\section{- $\quad$ Ziziphus lotus (L.) Lam}

$\mathrm{Ph}(\mathrm{Nph})$ - zones non forestiers ; plaines, basses et moyennes montagnes SA SH - Tout le Maroc et ne dépasse pas 1350m ;

\section{RUTACEAE}

\section{- $\quad$ Ruta L.}

- $\quad$ Ruta montana $L$.

Ch - forêts claires, matorrals rocailleux ; basses et moyennes montagnes A SA SH H - AA HA MA Man Mam Op Om LM R - localité : Toute la zone d'étude au niveau des basses et moyennes montagnes ;

ROSACEAE

\section{Crataegus $L$.}


- $\quad$ Crataegus oxyacantha L. (Aubépine)

(Ph)(Nph- SA SH H ; Basses et moyennes montagnes HA MA Mam Op Om LM R- large distribution au niveau de la zone d'étude ;

- Crataegus laevigata Poir.

(Ph)(Nph) - SA SH H ; Basses et moyennes montagnes ; HA MA Mam Op Om LM R.

- $\quad$ Rosa L.

- $\quad$ Rosa sempervireus $L$.

$\mathrm{Ph}$ - Forêts et matorrals fraiches des plaines et des basses montagnes - A SA SH H - AA HA MA Mam Man Om LM R.

- Rubus L.

- $\quad$ Rubus ulmifolius Schott. (Ronce) (Tabghat)

Nph - haies, brossailles et ravains humides ; plaines, basses et moyennes montagnes - A SA SH H - Tout le Maroc sauf Ms et As ;

- $\quad$ Potentilla $L$.

- $\quad$ Potentilla reptans $L$.

H (G) - lieux humides, bords des eaux; plaines, basses et moyennes montagnes - A SA SH H - HA MA Mam Man Op Om LM R.

- Aphanes maroccana Haylander\& Rothm.

Th - Forêts, matorrals et ermes des montagnes - SH - MA Man R - localité : Aguelmouss (1250m), Had bouhssoussen (forêt sidi çalah ,1450m), Moulay Bouazza (1040m), Mbarkyene (820m); Al Go-Ida (1450m), Oulmès (1170m);

\section{OLEACEAE}

- $\quad$ Fraxinus L.

- $\quad$ Fraxinus angustifolia Vahl.

$\mathrm{Ph}$ - Basses et moyennes montagnes - SA SH H - HA MA Mam Man Om LM R.

- $\quad$ Phillyrea L. (amthal, el ktem)

- $\quad$ Phillyrea angustifolia $L$.

$\mathrm{Ph}$ - forêts claires et matorrals ; plaines, basses et moyennes montagnes - SA SH H - HA MA Man R - localité : Toute la zone d'étude ;

\section{- $\quad$ Phillyrea latifolia L.}

$\mathrm{Ph}$ - forêts claires et matorrals ; plaines, basses et moyennes montagnes - SA SH H - As AA HA MA Mam Man Om LM R - localité : Toute la zone d'étude ;

\section{- Olea L.}

- $\quad$ Olea europea L. (zebbouj)

$\mathrm{Ph}$ - plaines et basses montagnes - A SA SH H - Maroc non Saharien Toute la zone d'étude ;

SALICACEAE 
- $\quad$ Populus L.

- $\quad$ Populus alba L.

$\mathrm{Ph}$ - Bords des riviéres, lieux humides des plaines et des basses montagnes A SA SH H - As HA MA Mam Man Op LM R - localité : Mbarkyene $(820 \mathrm{~m})$; Al Go-Ida (1450m), Oulmès (1170m), Maaziz (520m), Tiddass (430m) ;

\section{- $\quad$ Populus nigra $L$.}

Ph - bords des eaux - SA SH H - Ms AA HA MA Man Op R- localité : Moulay Bouazza (1040m), Mbarkyene (820m); Al Go-Ida (1450m), Oulmès (1170m), Maaziz (520m), Tiddass (430m) ;

- $\quad$ Salix L.

- $\quad$ Salix pedicellata Desf.

$\mathrm{Ph}$ - bords des cours d'eaux, plaines et montagnes ne dépasse pas 1900m $\mathrm{SH} \mathrm{H}$ - Tout le Maoc sauf Ms - EM ;

\section{- Salix alba L.}

$\mathrm{Ph}$ - bords des eaux, lieux humides - A SA SH - HA MA Man R - localité : Oulmès (1170m), Maaziz (520m), Tiddass (430m);

\section{SAXIFRAGACEAE}

\section{- Saxifraga $L$.}

- $\quad$ Saxifraga dichotoma Willd.

$\mathrm{H}$ - roches et pâturages terreux des montagnes calcaires - SH H - HA MA Man- localité : Oulmès (1170m);

\section{- $\quad$ Saxifraga embergeri Maire.}

$\mathrm{Ch}$ - rochers basaltiques des basses et moyennes montagnes - SH - Manlocalité : Oulmès (1170m)- EM ;

\section{TAXACEAE}

- Taxus L.

\section{- Taxus baccata $L$.}

Ph-Ravins ombragées et forêts de moyennes montagnes - H MA Man R Oulmès (1170m), Maaziz (520m), Tiddass (430m) ;

\section{TAMARICACEAE}

\section{Tamarix $L$.}

- Tamarix canariensis Willd.

$\mathrm{Ph}$ - Oueds des plaines et basses montagnes - SA SH - Ms MA Mam Rlocalité : Oued Grou (820m) ; Al Go-Ida (1450m) ; Oued Bouhmael ;

\section{THYMELACEAE}

\section{- $\quad$ Thymelea Miller.}

- $\quad$ Thymelea argentata (Lam.) Pau

Ch - matorrals - SH - Man Om LM R - localité: Oulmès (1170m);

- $\quad$ Thymelea virgata (Desf.) Endl. 
Nph - forêts claires , matorrals, sol terreux et rocailleux ; plaines , basses et moyennes montagnes -SA SH H - HA MA Mam Man Op Om LM R localité : Aguelmouss (1250m) , Had bouhssoussen ( forêt sidi çalah ,1450m), Moulay Bouazza (1040m), Mbarkyene (820m); Al Go-Ida (1450m), Oulmès (1170m), Maaziz (520m), Tiddass (430m) - EMA ;

\section{URTICACEAE}

- Urtica L.

- Urtica caudata Vahl.

Th - Champs, décombres, plaines et montagnes - A SA SH H- HA MA Mam Man Op Om LM R ;

\section{- Urtica pilulifera $L$.}

Th - bords des chemins, haies, décombres ; nitrophiles, plaines et basses montagnes - A SA SH H- HA MA Mam Man Op Om LM R ;

- $\quad$ Urtica dioica $L$.

G - lieux cultivés humides, bords des seguias, ravins frais, nitrophiles - A SA SH - MA Mam Man ;

\section{UMBELLIFERAE}

- Ammi L.

- $\quad$ Ammi visnaga (L.) Lam

Th - jachères et cultures - SA SH H - Tout le Maroc

- $\quad$ Bupleurum L.

- $\quad$ Bupleurum rigidum $L$.

$\mathrm{Ch}$ - forêts claires, pentes pierreux sèches ; basses et moyennes montagnes SA SH - As HA MA Man - localité : Oulmès (1170m);

\section{- Bupleurum spinosum Gouan.}

Ch - basses, moyennes et hautes montagnes - SA SH H ; As AA HA MA Om R localité : Aguelmouss (1250m), Had bouhssoussen ( forêt sidi çalah ,1450m), Moulay Bouazza (1040m), Mbarkyene (820m); Al Go-Ida (1450m);

\section{- $\quad$ Carum L.}

\section{- $\quad$ Carum verticillatum (L.) Koch}

$\mathrm{H}$ - prairies humides, bords de ruisselets des montagnes - SH H - MA Man -localité : Oulmès $(1170 \mathrm{~m})$ et Ment $(1215 \mathrm{~m})$;

- $\quad$ Ferrula L.

- $\quad$ Ferrula communis $L$.

G - plaines, basses et moyennes montagnes - A SA SH H - Tout le Maroc ;

- Ferrula biumbellata Pomel.

G - plaines et basses montagnes - SA SH - Mam Man : localité : Jbel El harcha (930m);

- $\quad$ Eryngium L.

- $\quad$ Eryngium ilicifolium Lam. 
Th - clairières rocailleux des forêts sèches, steppes, pâturages désertique SA - Toutes les divisions du Maroc ;

\section{- $\quad$ Eryngium triquetrum Vahl.}

$\mathrm{H}$ - pâturages rocailleux, clairières des forêts, cultures ; plaines, basses et moyennes montagnes - A SA SH H - HA MA Mam Man Op Om LM R EM ;

\section{- $\quad$ Eryngium compestre $L$.}

$\mathrm{H}$ - clairières des forêts, pâturages rocailleux - A SA SH H - HA MA Mam Man Om LM R ;

\section{- $\quad$ Eryngium tricuspidatum L.}

H (G) - Matorrals, plaines, basses et moyennes montagnes - SA SH H - Ms AA HA MA Mam Man Om LM R.

\section{- $\quad$ Thapsia L.}

- Thapsia garganica Aut.

$\mathrm{G}$ - clairières des forêts, ermes sablonneux et rocailleux, steppes; plaines et basses montagnes - A SA SH H - HA MA Mam Man Op Om LM R ;

\section{- Thapsia transtagana Brot.}

G - Clairières des forêts, sablonneux et rocailleux, steppes - plaines et basses montagnes

SA SH H - HA MA Mam Man Op Om LM R.

\section{- Daucus tenuisectus Cosson ex Batt.}

Th - pentes rocailleuses des basses et moyennes Montagnes siliceuses - SA SH H; AA (Ida-ou-Gnidif ; Aït Baha) HA MA Man - localité : large répartition dans la zone d'étude ;

- Magydaris pastinacea (Lam.) Paol.

G - ravins et vallons humides, bords de ruisseaux, plaines et basses montagnes - SA SH - MA Man Om LM R - localité : Aguelmouss (1250m) , Had bouhssoussen ( forêt sidi çalah ,1450m), Moulay Bouazza (1040m), Mbarkyene (820m); Al Go-Ida (1450m), Oulmès (1170m), Maaziz (520m), Tiddass(430m);

\section{SCROPHULARIACEAE}

\section{- $\quad$ Linaria $L$.}

- $\quad$ Linaria micrantha (L.) Desf.

Th - champs, pâturages rocailleux, graviers des rivières, clairières des forêts ; plaines et basses montagnes - A SA SH H - AA HA MA Mam Man Op Om LM R ;

\section{- $\quad$ Linaria latifolia Desf.}

Th - champs et matorrals des terrains argileux ; plaines et basses montagnes - A SA SH - MA Mam Man LM R ;

\section{- $\quad$ Linaria viviesiae Emberger.}


Th - rocailles arides ; basses montagnes siliceux - SA SH - Man - localite : Oulmès $(1170 \mathrm{~m})$ - EM ;

- $\quad$ Verbascum L. = Celsia L.

- Verbascum sinuatum $\mathbf{L}$.

Th - $(\mathrm{H})$ - matorrals, ermes, steppes ; plaines, basses et moyennes montagnes - A SA SH H - Tout le Maroc ;

- Verbascum zaianensis (Murb).

= Celsia zaianensis (Murb).

$\mathrm{H}$ - lieux pierreux et matorrals des basses montagnes - SH - MA Man localité : Aguelmouss (1250m) , Had bouhssoussen ( forêt sidi çalah ,1450m), Moulay Bouazza (1040m), Mbarkyene (820m); Al Go-Ida (1450m), Oulmès (1170m), Maaziz (520m), Tiddass(430m) ,Jbel El harcha( 930m) - EM ;

\section{VERBENACEAE}

Vitex $L$.

- Vitex agnus castus $L$.

Nph - bords des oueds ; plaines et basses montagnes - SA SH H - Tout le Maroc ;

\section{VIOLACEAE}

\section{- Viola L.}

- Viola arborescens $L$.

Ch - forêts clairs, rochers, pâturages pierreux et sablonneux du littoral et des basses montagnes - SA SH - Mam Man Om LM R ;

\section{ZYGOPHYLLACEAE}

\section{- $\quad$ Fagonia L.}

- $\quad$ Fagonia cretical L.

Ch - forêts claires, matorrals, rochers, rocailles; plaines et basses montagnes - A SA SH - Tout le Maroc ;

- Peganum L.

- $\quad$ Peganum harmala $L$.

Ch - steppes, champs incultes, décombres ; plaines et basses montagnes SA - Tout le Maroc

\section{- Tribulus L.}

\section{- $\quad$ Tribulus terestris $L$.}

Th - bords des chemins, lieux herbeux sablonneux; plaines et basses montagnes - SA SH - Ms HA MA Mam Man Op LM R ;

\section{Conclusion}

L'analyse floristique de la région étudiée a permis de deceler que parmi les 360 espèces végétales recencées, 46 sont des Fabacées, 44 des Astéracées, 32 des Caryophyllacées, 19 des Lamiacées. Ces familles 
comptent 43,9\% de l'ensemble de la flore de la région étudiée, le reste des espèces appartient à 48 autres familles qui comptent 56,10\%.

Selon le catalogue des plantes vasculaires rares, menacées ou endémiques du Maroc, établi par Fennane et Ibn Tatou en 1998: - Trois espèces sont très rares, il s’agit de : Calendula arvensis, Juncus bryonius et stachys circinnata; Six espèces rencontrées dans la région d'étude sont endémiques de la région Maroc-Algérie; il s’agit de: Asparagus altissimus, Crepis pulchra, Silene patula, Silene pomellii, Rhamnus lycoides et Thymelea virgata.

Quatre espèces sont des endémiques partagées entre le Maroc, l'Algérie et la Tunisie; il s’agit de: Asphodelus acaulis, Helianthemum ledifolium, Ballota hirsuta et Reseda lutea;

Une espèce est connue comme étant endémique du Maroc et de la péninsule Ibérique; il s’agit d’Andryala arenarea.

Quarante trois espèces rencontrées dans la région d'étude sont endémiques du Maroc; il s’git de: Andryala laxiflora, Andryala integifolia, Echium humile, Calliyriche mathezzi, Arenarea emerginata, Polycarpon sauvagei, Silene hirsutum, Silene mentagensis, Sedum modestum, Sedum jahandiezzi, Convolvulus leucochnous, Helianthemum lexifolium, Euphorbia clementei, Euphorbia rimarum, Fumaria rupestris, Erodium sebaceum, Erodium chium, Lavandula stoechas, Teucrieum decipiens, Teucrieum zaianum, Stachys arenaria, Stachys zaiana, Genista hirsuta, Lotus weilleri, Lotus marrocanus, Polygonum aviculare, Salix pedicellata, Thymelea virgata, Eryngium triquetrum, Linaria viviesiae, Celsia zaianensis, Paronychia capitata, Asphodelus gracilis, Anacyclus clavatus, Centeurea pullata, Tolpis barbata, Alyssum simplex, Anthyllis vulmeraria, Genista scorpius, Cytisus grandiflorus, Juncus bufonius, Marrubium echinatum et Reseda alba;

Le spectre biologique est un spectre typique de l'ambiance bioclimatique semi-aride, avec un pourcentage de 49,50\% pour les thérophytes, 6,66\% pour les géophytes, $20 \%$ pour les hémicryptophytes, $10 \%$ pour les chaméphytes, $6,11 \%$ pour les nanophanérophytes et $7,8 \%$ pour les phanérophytes.

L’aridité du milieu a favorisé un net développement des théropytes aux dépens d'autres types biologiques.

Dans l'étage bioclimatique subhumide, on rencontre le maximum d'espèces avec plus de $60 \%$ de la flore totale, les caractéristiques pluviothermiques moyennes de cet étage expliquent sa très forte richesse floristique. 


\section{References:}

Aafi A., Sghir M. \& Fechtal M. (2004). Espèces remarquables de la flore du Maroc : 146p.

Bammi, 2002. Etude de la diversité floristique et valorisation des plantes médicinales de la forêt de l'Achach (plateau central).Thèse de doctorat National. Université Ibn Tofail, Fac. des Sciences, Kénitra, Maroc, 278p.

Beaudet G., 1969. Le plateau central marocain et ses bordures: étude géomorphologique. Inframar, Rabat .478p.

Combe M., Ferre M., Thauvi J., 1975. Ressource en eau du Maroc (tome 2), plaines et bassins du Maroc Atlantique (meseta central et meseta côtière), note de mémoire du service de géologie N²31. p146- p17.

Dahmani.A., 1995. Développement des auréoles de contact d'Oulmès et de Ment (Maroc Central): Etendue, zones métamorphique et histoires de réchauffement et de refroidissement $11.27 \mathrm{p}$.

Daoudi A, Zerkani S, Nassiri L, et al. (2013) Inventaire des plantes médicinales de la commun d'Agelmouss - Province de Khénifra-Maroc. Science Lib; Editions Mersenne 5, n¹31012. 19 p

Emberger L., 1932.Sur une formule climatique et ses applications en Botanique. La météorologie, $10 \mathrm{p}$.

Emberger, L. \& R. Maire (1941). Catalogue des Plantes du Maroc. [vol. 4] Minerva éd., Alger. Supplément général, vol. 1, 2 \& 3. [p.1045]

Fennane M. \& M. Ibn Tattou (1998). Catalogue des plantes vasculaires rares, menacées ou endémiques du Maroc. Bocconea 8: 1-243, Palermo.

Fennane M., M. Ibn Tattou, J. Mathez, A. Ouyahya \& J. El Oualidi (1999). Flore Pratique du Maroc, vol. 1. Trav. Inst. Sci., sér. Bot. 38. 558pp.

Fennane M. \& M. Ibn Tattou. (2005). Flore vasculaire du Maroc : Inventaire et Chorologie, vol. 1, Trav. Inst. Sci., sér. Bot. 37: 483p.

Fennane M., M. Ibn Tattou, A. Ouyahya. \& J. El Oualidi. (2007). Flore Pratique du Maroc, vol. 2. Trav. Inst. Sci., sér. Bot. 38. 636p.

Hammoudi, A. (2002). La Subéraie : Biodiversité Et Paysage, Rapport Présenté Au Colloque Vivexpo Inst. Médit. Du Liège. France., Juin, 2002. $5 p$.

M'hirit.O et Philippe. B \& al (1999). le grand livre de la forêt Marocaine, Editions Mardaga, 1999 - 280 pages.

El Oualidi J \& al (2012).Checklist des endémiques et spécimens types de la flore vasculaire de l'Afrique du Nord. Document de l'institut scientifique, $\mathrm{N}^{\circ} 25,2012$. 189p.

Sauvage Ch., 1961. Recherches géobotaniques sur les subéraies marocaines.Trav.Inst.Chér. Série bot.21, Rabat, 462 p.

Quezel P., Santa S., 1962. Nouvelle flore de l'Algérie et des Régions désertiques méridionales, Tome I. Edition centre national de la recherche scientifique. 565p. 
Quezel P., Santa S., 1962. Nouvelle flore de l’Algérie et des régions désertiques méridionales, Tome II. Edition centre national de la recherche scientifique. p571-p1165. 